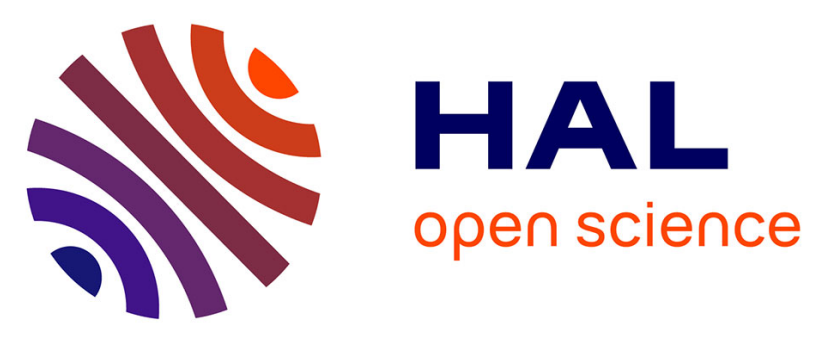

\title{
Plasticité musculaire et traitement physique dans la parésie spastique déformante : physiopathologie de la sous-utilisation et réversibilité par le réentrainement intensif
}

I. Npochinto Moumeni, Y. Njankouo Mapoure, J.M. Gracies, Emmanuel

Moyse, M. Temgoua, J.P. Moulangou, N.A.-N. Moumeni

\section{To cite this version:}

I. Npochinto Moumeni, Y. Njankouo Mapoure, J.M. Gracies, Emmanuel Moyse, M. Temgoua, et al.. Plasticité musculaire et traitement physique dans la parésie spastique déformante : physiopathologie de la sous-utilisation et réversibilité par le réentrainement intensif. NPG: Neurologie - Psychiatrie Gériatrie, 2021, 21 (124), pp.227-242. 10.1016/j.npg.2021.03.003 . hal-03332365

\section{HAL Id: hal-03332365 https://hal.science/hal-03332365}

Submitted on 2 Sep 2021

HAL is a multi-disciplinary open access archive for the deposit and dissemination of scientific research documents, whether they are published or not. The documents may come from teaching and research institutions in France or abroad, or from public or private research centers.
L'archive ouverte pluridisciplinaire HAL, est destinée au dépôt et à la diffusion de documents scientifiques de niveau recherche, publiés ou non, émanant des établissements d'enseignement et de recherche français ou étrangers, des laboratoires publics ou privés. 


\section{NPG Neurologie - Psychiatrie - Gériatrie \\ Plasticité musculaire et traitement physique dans la parésie spastique déformante: physiopathologie de la sous- utilisation et réversibilité par le réentrainement intensif --Projet de manuscrit--}

\begin{tabular}{|c|c|}
\hline Numéro du manuscrit: & NPG-D-21-00016 \\
\hline Type d'article: & Article original \\
\hline Mots-clés: & $\begin{array}{l}\text { Accident Vasculaire Cérébral; Parésie spastique déformante; neurorééducation } \\
\text { intensive; thérapie par contrainte induire; Contrat d'Auto-rééducation Guidé }\end{array}$ \\
\hline Auteur correspondant: & $\begin{array}{l}\text { Ibrahim NPOCHINTO MOUMENI, gérontologue, rééducateur fonctionnel } \\
\text { Hôpital Raymond-Poincare: Hopital Raymond-Poincare (laboratoire handicap } \\
\text { neurlocomoteur; physiopathologie, biothérapie et pharmacologie appliquée, ISERM } \\
\text { 1179, UMR END-INCAP } \\
\text { FRESNES, ile de france FRANCE }\end{array}$ \\
\hline Premier auteur: & Ibrahim NPOCHINTO MOUMENI, PhD \\
\hline \multirow[t]{7}{*}{ Ordre des auteurs: } & Ibrahim NPOCHINTO MOUMENI, PhD \\
\hline & yacouba Njankouo Mapoure \\
\hline & Emmanuel Moyse, chef de laboratoire de génétique de l'univer tours \\
\hline & jean michel gracies, chef service neurorééducation \\
\hline & Michael TEMGOUA, neurologue, hôpital bethsada \\
\hline & jean pierre MOULANGOU MOULANGOU, médecin généraliste \\
\hline & Abdel-Nasser Njikam MOUMENI, kinésithérapeute \\
\hline Résumé: & $\begin{array}{l}\text { Un des problèmes majeurs des patients ayant fait un accident vasculaire cérébral } \\
\text { (AVC) est la déformation corporelle. Ces patients acceptent plus facilement d'avoir } \\
\text { une motricité et une fonctionnalité diminuées que d'avoir un corps déformé. Ils ne } \\
\text { consultent pas en raison de la spasticité mais ils viennent se plaindre de raideur, } \\
\text { déformations, limitations des capacités fonctionnelles, inconfort, et/ou douleur. } \\
\text { L'objectif principal de la réadaptation après un AVC est de restaurer l'indépendance } \\
\text { des patients dans leurs activités de vie quotidienne. S'il est vrai que les AVC laissent } \\
\text { un contexte de vulnérabilité motrice de type parésie, il n'en est pas moins vrai que la } \\
\text { sous-utilisation aggrave cet état et initie une autre pathologie locale sous-jacente, la } \\
\text { myopathie spastique, qui apparaît dès les premiers jours post-AVC, et centrale, la } \\
\text { dégénérescence neuronale, par une réorganisation des circuits afférents (plasticité mal } \\
\text { adaptative) qui augmente la déficience motrice primaire. Ce mécanisme entraîne la } \\
\text { parésie spastique déformante qui est réversible à partir du moment où l'on commence } \\
\text { à utiliser activement le côté lésé. Les traitements par les exercices ou les thérapies } \\
\text { physiques sont plus efficaces que les traitements pharmacologiques lorsqu'il s'agit de } \\
\text { la récupération motrice, du maintien ou des adaptations des capacités fonctionnelles } \\
\text { des personnes en générale, et des cérébrolésés en particulier. C'est pourquoi il est } \\
\text { nécessaire, à côté des traitements pharmacologiques, de proposer une quantité plus } \\
\text { importante de traitements physiques focaux des muscles cibles (les antagonistes) par } \\
\text { des techniques d'étirement agressif, prolongé, activo-dynamique et des mouvements } \\
\text { alternatifs d'amplitude maximale, tant en cabinet avec le patient, qu'à domicile (via un } \\
\text { contrat d'auto-rééducation guidée et un registre) afin de non seulement briser le cercle } \\
\text { néfaste de la sous-utilisation acquise après un AVC, mais aussi de vaincre ces } \\
\text { déformations corporelles inesthétiques qui créent parfois du désespoir dans la vie } \\
\text { sociale et professionnelle de ces patients. }\end{array}$ \\
\hline
\end{tabular}


Plasticité musculaire et traitement physique dans la parésie spastique déformante : physiopathologie de la sous-utilisation et réversibilité par le réentrainement intensif

Muscle plasticity and physical therapy in deforming spastic paresis: physiopathology of underuse and reversibility byintensive retraining

Auteurs:

NPOCHINTO MOUMENI Ibrahim ${ }^{1,2,3,4,5}$, Yacouba Njankouo Mapoure ${ }^{6}$, Jean Michel Gracies ${ }^{2}$, Emmanuel Moyse ${ }^{7}$,TEMGOUA Michael ${ }^{4}$, MOULANGOU Jean Pierre ${ }^{8}$, Njikam MOUMENI AbdelNasser ${ }^{9}$

1- Laboratoire handicap neuromusculaire ; physiopathologies, biothérapie et pharmacologie appliquée ; U1179 (END-ICAP)-INSERM / Université de Versailles, Hôpitaux Universitaire Raymond Poincaré, Garches.

2- $\quad$ Laboratoire Analyse et Restauration du Mouvement, service de rééducation neuro locomotrice et ostéoarticulaires, Hôpitaux Universitaires Henri-Mondor EA 7377 BIOTN, Université Paris-Est Créteil 51, avenue du Maréchal deLattre de Tassigny 94010 Créteil France

3- $\quad$ Faculté de médecine Sorbonne université, Paris 6 (Université Pierre et Marie Curie), Hôpitaux Universitaire Pitié-Salpêtrière

4- Institut des Neurosciences appliquées et de Rééducation Fonctionnelle (INAREF) Yaoundé (Cameroun)Messamendongo-Odza.

5- $\quad$ Faculté des sciences de la santé et de psychologie de Bircham International University, Madrid-Espagne

6- $\quad$ Faculté de médecine de de science pharmaceutique de l'université de DoualaCameroun

7- Département de biologie et de génétique UMR Université de Tours-CNRS INRA 7247 - Physiologie de la Reproduction et desComportements (PRC) Centre de Tours 37380 Nouzilly Parc de Grandmont 37200 Tours 6 France.

8- $\quad$ Centre Hospitalier sud Francilien, Paris, France

9- $\quad$ Centre médical de rhumatologie et de kinésithérapie Saint Israël, Douala (Cameroun)

Correspondance : NPOCHINTO MOUMENI Ibrahim (moumeniibrahim@yahoo.fr) 


\section{Plasticité musculaire et traitement physique dans la parésie spastique déformante : physiopathologie de la sous-utilisation et réversibilité par le réentrainement intensif}

Muscle plasticity and physical therapy in deforming spastic paresis: physiopathology of underuse and reversibility byintensive retraining

Auteurs:

NPOCHINTO MOUMENI Ibrahim ${ }^{\mathbf{1 , 2 , 3 , 4 , 5}}$, Yacouba Njankouo Mapoure ${ }^{\mathbf{6}}$, Jean Michel Gracies ${ }^{2}$, Emmanuel Moyse ${ }^{7}$,TEMGOUA Michael ${ }^{4}$, MOULANGOU Jean Pierre ${ }^{8}$, Njikam MOUMENI Abdel-Nasser ${ }^{9}$

1- Laboratoire handicap neuromusculaire ; physiopathologies, biothérapie et pharmacologie appliquée ; U1179 (END-ICAP)-INSERM / Université de Versailles, Hôpitaux Universitaire Raymond Poincaré, Garches.

2- Laboratoire Analyse et Restauration du Mouvement, service de rééducation neuro locomotrice et ostéoarticulaires, Hôpitaux Universitaires Henri-Mondor EA 7377 BIOTN, Université Paris-Est Créteil 51, avenue du Maréchal deLattre de Tassigny 94010 Créteil France

3- Faculté de médecine Sorbonne université, Paris 6 (Université Pierre et Marie Curie), Hôpitaux Universitaire Pitié-Salpêtrière

4- Institut des Neurosciences appliquées et de Rééducation Fonctionnelle (INAREF) Yaoundé (Cameroun)Messamendongo-Odza.

5- Faculté des sciences de la santé et de psychologie de Bircham International University, Madrid-Espagne

6- Faculté de médecine de de science pharmaceutique de l'université de Douala- Cameroun

7- Département de biologie et de génétique UMR Université de Tours-CNRS INRA 7247 Physiologie de la Reproduction et desComportements (PRC) Centre de Tours 37380 Nouzilly Parc de Grandmont 37200 Tours 6 France.

8- Centre Hospitalier sud Francilien, Paris, France

9- Centre médical de rhumatologie et de kinésithérapie Saint Israël, Douala (Cameroun)

Correspondance : NPOCHINTO MOUMENI Ibrahim (moumeniibrahim@yahoo.fr) 


\section{Résumé}

Un des problèmes majeurs des patients ayant fait un accident vasculaire cérébral (AVC) est la déformation corporelle. Ces patients acceptent plus facilement d'avoir une motricité et une fonctionnalité diminuées que d'avoir un corps déformé. Ils ne consultent pas en raison de la spasticité mais ils viennent se plaindre de raideur, déformations, limitations des capacités fonctionnelles, inconfort, et/ou douleur. L'objectif principal de la réadaptation après un AVC est de restaurer l'indépendance des patients dans leurs activités de vie quotidienne. S'il est vrai que lesAVC laissent un contexte de vulnérabilité motrice de type parésie, il n'en est pas moinsvrai que la sous-utilisation aggrave cet état et initie une autre pathologie locale sousjacente, la myopathie spastique, qui apparaît dès les premiers jours post-AVC, et centrale, la dégénérescence neuronale,par une réorganisation des circuits afférents (plasticité mal adaptative) qui augmente la déficience motrice primaire. Ce mécanisme entraîne la parésie spastique déformante qui est réversible à partir du moment où l'on commence à utiliser activement le côté lésé. Les traitements par les exercices ou les thérapies physiques sont plus efficaces que les traitements pharmacologiques lorsqu'il s'agit de la récupération motrice, du maintien ou des adaptations des capacités fonctionnelles des personnes en générale, et des cérébrolésés en particulier. C'est pourquoi il est nécessaire, à côté des traitements pharmacologiques, d e propos e r une quantité plus importante de traitements physiques focaux des muscles cibles (les antagonistes) par des techniques d'étirement agressif, prolongé, activo-dynamique et des mouvements alternatifs d'amplitude maximale, tant en cabinet avec le patient, qu'à domicile (via un contrat d'auto-rééducation guidée et un registre) afin de non seulement briser le cercle néfaste de la sousutilisation acquise après un AVC, mais aussi de vaincre ces déformations corporelles inesthétiques qui créent parfois du désespoir dans la vie sociale et professionnelle de ces patients.

Mots-clés : Accident Vasculaire Cérébral ; Parésie spastique déformante ; neurorééducation intensive ; thérapie par contrainte induire ; Contrat d'Auto-rééducation Guidé.

\section{Summary}

One of the biggest problems for our stroke patients is the deformity of their body. The patient more easily accepts having reduced motor skills and functionality than having a deformed body. They do not often consult for reasons of spasticity, they come to complain of stiffness, deformities, limitations in functional abilities, discomfort, and / or pain. The main goal of stroke rehabilitation is to restore patients' independence in their activities of daily living (ADL), and, alongside, their health-related quality of life (HR-QoL). While it is true that strokes leave a context of motor vulnerability of the paresis type, it is nevertheless true that underutilization aggravates this state, and initiates another underlying local pathology, spastic myopathy, which appears as early as the first days after astroke; it also initiates neural degeneration via a reorganisation of the related circuits (mis-adaptive plasticity) which increases the primary motor impairment. This mechanism leads to deforming spastic paresis, which is reversible as 
soon as the impaired side is reactivated. Exercise or physiotherapy treatments are more effective than pharmacological treatments when it comes to motor recovery, maintenance or adaptation of people's functional abilities in general, particularly in case of brain damage. It is thus important, alongside pharmacological treatments, to offer focal physical treatment targeting the appropriate muscles (the antagonists) using techniques of aggressive, prolonged stretching, also entailing alternative movements of maximum amplitude, both in consultation with the patient and at home (via a guided self-education contract and register) in order not only to break the vicious cycle of underuse acquired after a stroke, but also to overcome the bodily distortions that sometimes create despair in the subject's social and professional life.

Keywords: Stroke; spastic paresis; intensive neurorehabilitation; constraint-induced therapy; guided selfrehabilitation contract. 


\section{Introduction}

Environ 25,7 millions de personnes dans le monde font un accident vasculaire cérébral (AVC) chaque année, avec environ 2/3 de survivants et 6,5 millions de décès liés à l'AVC ou à ses complications (entre les $1^{\text {ers }}$ jours et la $1^{\text {ère }}$ année post-AVC), faisant de l'AVC la deuxième cause de décès et l'une des principales causes de handicap acquis chezl'adulte. Le déficit le plus fréquent est l'hémiparésie [1,2,3]. Au sujet de la récupération globale, de nombreuses études cliniques ont souligné depuis longtemps, de remarquables capacités de récupération des patients victimes d'AVC [4,5]. Ces études ont permis de bien objectiver l'amélioration des déficiences motrices, ainsi que la chronologie au cours des six premiers mois suivant l'AVC. La parésie spastique apparaît plus tard par le phénomène de sous/hypo-utilisation avec plusieurs gênes fonctionnelles et psychologiques (tableau 1), comportant une parésie de l'agoniste et une hyperactivité de l'antagoniste. Cependant, une seconde affection d'ordre musculaire, la myopathie spastique [6,7], apparaît rapidement, souvent même dès les premiers jours après la période dite flasque. La sous-utilisation en position courte de certains muscles dans le contexte d'une parésie des muscles opposés, s'accompagne d'une perte de leur tension longitudinale. Cette perte de tension est la première étape d'une série de transformations structurelles, biomécaniques, puis physiologiques des muscles sous-utilisés, incluant entre autres une perte de leur extensibilité, de leur longueur et une augmentation du collagène non extensible. Aux stades subaigus, puis chroniques du syndrome, les affections neurologiques et musculaires coexistent ensemble et semblent même s'entretenir mutuellement [6,8,9]. Meige [10] en 1905, parlait déjà de l'amnésie fonctionnelle motrice. L'oubli psychologique de ces patients AVC fait que non seulement ils oublient d'employer leur main lésée, mais ils développent une hyperutilisation de la main saine, majorant ainsi le déséquilibre hémisphérique post-lésion [11]. Cette approche est l'élément initial de base de la technique de la thérapie par contrainte induite (TCI) de Taub [12,13].

Tardieu et al [14] et Tabary et al [15], en 1972, parlaient déjà de l'influence de l'immobilisation sur la pathologie neurologique juste après quelques heures dans le modèle animal. Ces auteurs ont observé une contracture myotatique, caractérisée par un changement de la tension passive, sur les courbes d'allongements transversaux des muscles. Ces changements sont associés à une diminution considérable du nombre de sarcomères, et un spasme tétanique observé à l'EMG (électromyographie) sur les soléaires. Ces phénomènes avaient aussi été observés auparavant [16,17]. Tous ces auteurs décrivent une augmentation du taux collagénique et du tissu conjonctif, responsables de l'atrophie et de la raideur, avec une diminution de $37 \%$ de taux fractionné de synthèse protéique (le $14^{\text {ème }}$ jour de l'immobilisation) et de $30 \%$ de la perte de la masse musculaire. Un épaississement myofacial avait été aussi observé, ainsi qu'une augmentation de la proportion de la graisse inter et intramusculaire. Avec une augmentation du contenu en ARNm (Acide RiboNucléique messager) pour des composants du système Ubiquitin-proteasome, associée à une baisse 
de $15 \%$ de force musculaire déjà à la deuxième heure d'immobilisation, et une résistance à un étirement passif à partir de la 18ème heure d'immobilisation [14,15,16,17].

Aux vues de la micro et neuro biologie [11], la maladie musculaire créée par l'immobilisation est ultra-précoce : avec tout d'abord une dominante métabolique, qui devient mécanique, puis suit alors un phénomène de réorganisation dite «plasticité mal adaptative » qui modifie aussi la neurophysiologie. Il est ainsi observé l'impact négatif de l'immobilisation en position courte en laboratoire neurobiologique sur le modèle animal, qui donne naissance à la diminution de la tension musculaire (unloading ; décharge), qui est le premier mécanisme de la contracture musculaire, puis une accumulation de graisse, un stress oxydatif et une accumulation du tissu conjonctif et un épaississement des membranes (figure1 et 2).

Tous ce désordre neurophysiologique entraîne une atrophie et une raideur musculaire avec une réponse exagérée à l'étirement et une augmentation des réponses spinales (réaction à un afférent donné). Le muscle étant moins extensible, les forces d'un étirement donné sont transmises sans être amorties aux fuseaux neuro-musculaires (FNM) qui répondent de façon appropriée : envoi d'un message augmenté à la moelle épinière (ME) qui répond de façon appropriée, ce qui est même la conséquence et l'identité de la spasticité (figure 2). Une des causes de la spasticité est la réponse excessive à la stimulation du FNM par un muscle moins extensible et plus élastique. Ce phénomène de raideurs, d'atrophie, de perte de sarcomères, et de force musculaire (myopathie spastique déformante) est réversible à partir du moment où l'on lève l'immobilisation et commence à utiliseractivement le membre sous-utilisé/immobilisé [9,11,13-17]. Mais le fait de ne pas utiliser la voie cortico-spinale chez ces patients crée d'autres problèmes : le faisceau corticospinal permet une gradation du mouvement. Les autres faisceaux (rubrospinal, vestibulospinal, tectospinal, etc.) très utilisés par certaines espèces animales (amphibiens, batraciens, oiseaux) donnent des mouvements complets de type «tout ou rien ». Par contre, le sujet humain parétique se repose beaucoup sur le faisceau vestibulospinal pour produire le mouvement qui est sensible à la position puisqu'elle a une influence sur l'activitédu vestibule et sur la force de contraction qui est supérieure en position assise. Mais ces faisceaux ont très peu de capacité de mise au repos. La capacité de repos du sujet sain non entraîné n'est pas totale (environ 2\% d'activité, bien qu'étant en «repos»). Chez le sujet parétique, la capacité de repos est inexistante. Leurs faisceaux du tronc cérébral ont en effet une activité ionique transmembranaire permanente (continue au travers des contractions/co-contractions, et de l'hyperactivité de l'antagoniste qui pèse de tout son poids sur l'agoniste et le met en position fléchie avec déformation) et dystonique. Le muscle dystonique est contracté en permanence, ce qui entraîne donc sa rétraction (et sa dégradation locale microbiologique avec la myopathie spastique), et si l'on stimule trop ce muscle spastique sans faire d'étirements compensatoires, il raccourcit davantage [6,7,8,9] (c'est pourquoi l'étirement doit absolument précéder le renforcement chez les hémiparétiques). Le deuxième problème de ces patients parétiques est celui décrit depuis longtemps par Sherrington (1898) et Babinski (1912) [18,19] : c'est la rétraction qui augmente l'hyperactivité musculaire (et non l'hyperactivité qui augmenterait la rétraction) entraînantelle-même la rétraction de l'agoniste qui a trop de difficultés face à la tension de l'antagoniste, avec déséquilibre intermusculaire (figure 3). Ces travaux ont conduit à la démonstration que la spasticité était associée à une 
hyperexcitabilité en réponse à l'étirement lié à la perte des influences inhibitrices de la voie descendante (supraspinale). Cet article se propose de décrire la physiopathologie de la parésie spastique, puis celle de la sous-utilisation et son influence sur la lésion neurologique (AVC), puis l'effet de la thérapie induite par la contrainte, les valeurs cliniques de l'intensité, et enfin les techniques de neurorééducations avancées, employées dans notre service de neurorestauration du mouvement.

\section{Physiopathologie}

La spasticité est un désordre moteur caractérisé par une augmentation vitesse-dépendante du réflexe tonique d'étirement, et par une augmentation des réflexes ostéotendineux, résultant d'une hyperexcitabilité du réflexe d'étirement dans le cadre d'un syndrome pyramidal [17,18] (figure1). La spasticité est un symptôme fréquemment retrouvé chez les patients victimes de lésions du système nerveux central (AVC). Elle peut être utile dans une compensation de la perte de force motrice durant la marche, la course ou la posture, mais peut également devenir nocive avec des pertes fonctionnelles irréductibles et des contractures (tableau 1, figure 4). En général les patients ne consultent pas en raison de la spasticité mais ils viennent se plaindre de la raideur et des déformations (tableau 1, figure 3), des limitations des capacités fonctionnelles, de l'inconfort ou de la douleur. Le clinicien doit essayer de comprendre ce que les patients entendent par le terme «rigidité (spasticité) ». Dans toute discussion sur la gestion de la spasticité, une terminologie commune est importante pour reconnaitre les deux composants principaux de la raideur musculaire. Le premier est un raccourcissement dynamique des muscles qui est causé par la spasticité et le second est un raccourcissement fixe des muscles qui est décrit comme une contracture.

Le syndrome de la parésie spastique est d'abord un problème de l'antagoniste, c'est avant tout lui qui limite le mouvement. Tardieu et al [14] ont été les premiers à décrire ce principe de la réponse exagérée des muscles à leurs propres étirements qui dépend de la vitesse de l'étirement [15,20,21]. C'est un double syndrome, une sorte de double affection. Ces deux maladies affectent deux organes : le muscle et le système nerveux. Le désordre musculaire se traduit par une rétraction des tissus mous («myopathie spastique » décrite par de Gracies [7,8] : «la myopathie spastique et la rétraction des tissus mous avoisinants, et les différentes formes d'hyperactivité musculaire telles que la spasticité et la co-contraction spastique »), avecure diminution de la longueur, associée à une diminution de l'extensibilité observée sur le plan clinique. Mais le 
muscle produit également moins de force. Le désordre neurologique précède le désordre musculaire de quelques minutes/heures [14-17]. Il se caractérise par une parésie (agoniste) et une hyperactivité musculaire (antagoniste). La parésie n'est pas le phénomène le plus important du syndrome, mais la co-contraction en est un véritable problème, surtout lors de l'implémentation du mouvement volontaire [6-9,11]. La parésie spastique suit la perturbation chronique de l'exécution centrale de la commande volontaire. La fonction motrice chez les patients atteints de parésie spastique est soumise au fil du temps à un trépied fondamental, dont les deux derniers points sont évitables : (1) la lésion neuronale elle-même, qui provoque la parésie, c'està-dire la réduction du recrutement volontaire d'unités motrices ; (2) l'immobilisation, la sous-utilisation [10] relative de la partie du corps parétique, généralement imposée inconsciemmentpar l'environnement de soins (personnel de santé) actuel et le patient lui-même, qui provoque un raccourcissement adaptatif des muscles laissés dans une position raccourcie et une contracture articulaire [11], et (3) la désuétude chronique de la partie du corps parétique, qui est généralement auto-imposée chez la plupartdes patients $[9,11]$. La désuétude chronique provoque des arrangements plastiques dans les centres supérieurs qui réduisent encore la capacité de recruter volontairement des unités motrices, c'est-à-dire qui aggravent à nouveau la parésie de base qui est «l'influence de l'immobilisation sur la pathologie neurologique de Tardieu» [14,15]. Dans les stades subaigus et chroniques de la parésie spastique, la suractivité musculaire (spastique) sensible à l'étirement apparaît comme un troisième mécanisme fondamental de déficience motrice, avec la parésie et la contracture des tissus mous. On observe que la contracture musculaire est l'un des facteurs qui causent une réactivité excessive à l'étirement, ce qui aggrave à son tour la contracture. La réactivité excessive à l'étirement entrave également le recrutement volontaire des neurones moteurs, un concept appelé parésie extensible. Aucun de ces trois mécanismes de déficience (parésies, contracture et suractivité spastique) n’est réparti symétriquement entre les agonistes et les antagonistes. Ce qui génère un déséquilibre de couple autour des articulations et des déformations des membres. Conduisant ainsi à un déséquilibre nocif et progressif, mais qui peut être réversible par la démobilisation ou l'utilisation active [6-9,11,14,15].

\section{2- Comprendre la physiopathologie de la non/sous-utilisation}

D’après Meige [10], et son concept d'amnésie motrice, décrivant ce désordre moteur comme étant « 
distinct de la paralysie lésionnelle, secondaire à l'absence d'activité, liée à un phénomène de perte de mémoire fonctionnelle, qui peut être réversible. Et que la rééducation motrice devrait comporter des exercices répétés et prolongés de la fonction perdue »[10,11], l'atteinte laissée par l'AVC induit une certaine vulnérabilité (parésie) qui génère un phénomène de non-utilisation apprise qui empêche ou limite l'expression de la récupération motrice, compromettant ainsi les possibilités de récupération. Les lésions neurologiques importantes entraînent une dépression (diaschisis : dépression neuronale périlésionnelle et ipsilésionnelle) [22,23] importante dans la fonction motrice et/ou perceptuelle. Pendant cette période, le sujet ne peut pas utiliser parfaitement lecôté lésé. Les tentatives d'utiliser le membre affecté conduisent généralement à la douleur ou à une sur-demande élevée d'énergie, ou encore purement à l'échec. Ces conséquences douloureuses, difficiles et puissantes entraînent petit-à-petit la suppression, ou la sousutilisation du membre parétique.

Le sujet est tenté raisonnablement d'utiliser seulement le membre supérieur sain (ignorant le membre lésé du fait des échecs multiples). Il est donc récompensé négativement dans ce modèle de comportement, qui est auto-induit et renforcé par la parésie elle-même, qui n'est pas une véritable «paralysie », mais plus plutôt une diminution quantitative de la capacité de recruter volontairement et de manière synchrone les unités motrices [11]. En outre, après l'AVC, et probablement après la déafférentation, il y a une contraction marquée dans la taille de la représentation corticale du membre lésé qui est en corrélation avec le mouvement de ce membre atteint. Ceci n'est parfois pas satisfaisant pour le patient à l'échelle d'évaluation des activités de la vie quotidienne (AVQ). Ces processus interagissent pour produire une spirale descendante mal adaptative qui se traduit par « une non-utilisation apprise » du membre supérieur lésé, qui devient au fil du temps normale, mais aussi permanente. D'après cette théorie [10], la désafférentation conduit à une adaptation néfaste pour le membre supérieur lésé. Des circuits neuronaux corticaux et sous-corticaux avec un véritable cercle anormal, mal adaptatif. Le patient fait des tentatives infructueuses qui entraînent un renforcement négatif, lui-même à l'origine d'une répression comportementale et d'un masquage des capacités résiduelles qui sont à l'origine du phénomène de non ou sous-utilisation.

En parallèle, la diminution de la réalisation de mouvement entraîne une diminution des représentations corticales du mouvement qui va rendre encore plus difficile la réalisation des mouvements auto-entretenant ainsi le phénomène de non/sous-utilisation $[10,11]$. Ce phénomène est réversible dès la démobilisation etle réentraînement. S'il est vrai que l'AVC laisse un terrain de vulnérabilité motrice (parésie) d'une part, il est aussi vrai que la sous-utilisation, si elle n'est pas brisée de sitôt, favorisera une dégénérescence neuronale par une réorganisation des circuits afférents («plasticité mal adaptative ») [11] et augmentera la déficience et toute la cascade microneurobiologique [11,13-17].

Une étude sur l'animal dans notre laboratoire d'analyse du mouvement du CHU Henri Mondor [24] a montré que l'AVC crée une maladie musculaire par lui-même. C'est plus grave d'immobiliser ou de sous-utiliser un muscle sur le plan histologique, biomécanique, neurologique et presque pareil sur plan comportemental, puisque le même comportement de sous-utiliser ou refuser d'utiliser le membre lésé a été observé. Ce qui est en accord avec la notion d'amnésie motrice [10]. Au regard de cette étude, l'immobilisation en position 
courte est à proscrire, la sous-utilisation est à éviter. Par contre l'immobilisation en position longue a un avantage considérable et est recommandée chez les patients ayant fait un AVC dès la phase aiguë avant même la verticalisation $[11,24]$.

\section{3- Thérapie par la contrainte induite (TCI) ou Constraint Induced Movement Therapy (CIMT)}

Les principes de la TCI sont connus depuis longtemps. Tower [23] travaillant sur les singes (bien avant Taub et Wolf $[12,13])$ après section du faisceau pyramidal, avait remarqué que ces animaux se servaient de moins en moins de leurs membres supérieurs déficitaires. Aussi a-t-elle soupçonné l'existence d'une " non utilisation acquise » aggravant le déficit secondaire à la lésion. Elle disait : "l'initiative du mouvement est alors déléguée au côté sain, mais si le côté sain est contraint, le côté lésé avec une stimulation suffisamment importante, pourra être amené à agir ». Le port d'un système de contention bloquant l'utilisation du membre supérieur sain pendant quelques semaines entrainait immédiatement une utilisation beaucoup plus importante du membre déficitaire, et à plus long terme, cette amélioration fonctionnelle persistait clairement. Il faut reconnaitre que ces observations sont restées dans l'oubli jusqu'aux travaux de Taub et Wolf $[12,13]$. Dans l'hémiparésie spastique induite par l'AVC, au stade chronique, la fonction active du membre supérieur est particulièrement altérée, la proportion de patients recouvrant une main fonctionnelle n'étant que de $25 \%$. Ces limitations importantes restreignent les activités de la vie quotidienne des patients à de multiples niveaux, notamment social et professionnel, et pour cette raison, la TCI implique plus le membre supérieur. D'après certains auteurs, la porosité de la récupération du membre supérieur (MS) peut être liée au coefficient de faiblesse et de fatigabilité qui se trouve être plus élevé au MS qu'au MI. En outre, le coefficient de raccourcissement est plus élevé au MI qu'au MS, comme si la commande neurologique était de moins bonne qualité au MS qu'au MI [6-9].

Les premiers travaux sur la TCI ont concerné un nombre trop faible de patients, avec une méthodologie insuffisante (essais non randomisés, absence de contrôle en aveugle...) pour permettre de conclure formellement (à l'époque) sur l'efficacité de cette technique dans l'amélioration de la récupération après AVC. Avant 1950, aucune étude n'avait été publiée sur la TCI. En 1968, Knapp et Taub [13,14] reprennent le concept. Ils ont démontré initialement chez le primate [13,14], puis chez l'homme [25], l'influence de la non-utilisation ou sous-utilisation dans les troubles moteurs d'origine neurologique. La non-utilisation induite par la parésie génère un phénomène de non-utilisation apprise qui empêche ou limite l'expression de la récupération motrice, compromettant ainsi les possibilités de récupération. Cette technique(TCI) était utilisée par certaines équipes $[12,13,25,26]$ avec des variantes concernant la durée de port de la contrainte et de l'exercice associé. En 2006, la même équipe que celle qui a mise en évidence ce phénomène, a enfin démontré de façon non contestable, par une étude randomisée en simple aveugle avec suffisamment de patients, son intérêt dans l'utilisation du membre supérieur parétique dans les activités de la vie quotidienne (AVQ) avec maintien de l'effet à 24 mois[27]. Cependant, l'application à large échelle de cette technique est difficilement envisageable telle quelle, essentiellement du fait de la nécessité de 
pratiquer 6 heures de rééducation quotidienne en association au port de la contrainte.

Le principe général de la sous-utilisation apprise est qu'une certaine part du déficit d'utilisation du membre supérieur ne résulte pas des dommages directs sur des voies motrices, mais d'un phénomène comportemental compensatoire plurifactoriel, favorisant une sous-utilisation, voire une non utilisation du MS déficitaire.

Ce phénomène compensatoire s'explique par trois facteurs essentiels, à savoir : une plus grande difficulté à utiliser le MS déficitaire qu'à apprendre à se servir différemment du membre sain, des douleurs mécaniques du MS déficitaire gênant son utilisation et des troubles sensitifs (troubles proprioceptifs et superficiels) et sensoriels (négligence spatiale unilatérale). La TCI consiste à forcer l'utilisation du MS déficitaire, en immobilisant le MS sain, tout en entraînant de façon intensive le MS déficitaire. Cette technique a été le mieux évaluée, et son objectif est d'inverser l'apprentissage de la non/sous-utilisation, et de faciliter la réorganisation corticale dépendante de l'utilisation. Ces influences sont aussi perceptibles dans l'hémisphère lésé par un accroissement des cartes corticales dédiées au membre (lésé) contraint à son utilisation accrue qui conduit à un élargissement, dépendant de la quantité de l'utilisation de la représentation corticale de membre lésé, ce qui réduit l'effort dans son utilisation et fournit une base neuronale pour la rétention à long terme des gains réalisés en laboratoire ou en clinique tout en essayant de rééquilibrer la balance interhémisphérique du SNC [11].

Parmi les différents protocoles de TCI développés, celui proposé par Taub et al en 2006 [12-14,25-27], étant le seul ayant montré son intérêt dans une étude de qualité méthodologique suffisante [27], est considéré comme le gold standard de l'époque. Des méthodes alternatives ont également été étudiées [2831].Dans le protocole de référence de TCI, les patients doivent porter la contrainte durant $90 \%$ des heures de marche, entre le moment où ils se réveillent le matin et le moment où ils se couchent le soir. La contrainte est installée après la prise du petit déjeuner et la toilette, et est retirée pour l'élimination (selles et urines) et les repas en cas de nécessité absolue (Figure 5).

En conclusion, la TCI est significativement efficace. Le problème avec cette technique réside dans son côté exclusif (sa faisabilité par tous les patients et la disponibilité des thérapeutes: kinésithérapeute, ergothérapeute, enseignant en activité physique adaptée (APA), psychologue, etc.) à se focaliser sur l'observance d'un seul patient pour assurerson succès. Par ailleurs, la motivation du patient doit être de mise. La pratique de la TCI requiert certains prérequis cliniques : la capacité d'avoir au moins plus de $20^{\circ}$ d'extension du poignet et $15^{\circ}$ des doigts du côté lésé, la motivation du patient est fondamentale (cette motivation en clinique, s'observe surtout chez les patients jeunes, atteints du côté le plus employé. Lorsque c'est le bras gauche, le gaucher fera tout pour récupérer ce bras, lorsque c'est un droitier, il fera tout pour récupérer son bras droit. Le côté de la lésion et l'usage quotidien est essentiel à stimuler la motivation); l'absence de troubles cognitifs est aussi un critère majeur. L'intensité et la répétition sont des facteurs de la récupération motrice par apprentissage, même bien longtemps après l'AVC. Pour que la 
TCI soit efficace, l'on doit y associer de l'intensité, la répétition et surtout l'augmentation du temps de rééducation qui sont des paramètres majeurs à cette technique de TCI [11,32,33].

\section{4- Les travaux et connaissances sur l'intensité}

Pendant plusieurs générations, l'enseignement des stratégies « non intensives » a encouragé de façon parfois quasi dogmatique des équipes de rééducation à limiter le travail actif ou les exercices de renforcements dans la crainte de «réveiller la spasticité » ou de générer des douleurs [11,32-35]. Depuis les années 1990, de multiples études contrôlées ont cependant établi que le travail actif peut non seulement améliorer la fonction mais aussi réduire la spasticité, les rétractions musculaires et les douleurs $[33,36]$. Les éditoriaux des trois dernières décennies s'accordent sur l'intensité et le travail actif, et leurs donnent une place capitale parmi les moyens et méthodes d'optimisation de la récupération motrice d'une parésie après un AVC [11-13,32-37]. La rééducation du mouvement a été intensifiée en combinant deux éléments capitaux : le nombre de répétitions du mouvement à accomplir [11,32-37] et la difficulté dans l'accomplissement de chacun de ces mouvements [11-13,32,35-37]. Par conséquent, un mouvement difficile, répété de nombreuses fois correspond à une intensité optimale. [11-13,32,36].

Avant les années 1980, il n'existait que peu d'écrits sur la notion d'intensité d'entraînement rééducatif. On peut citer l'exemple de la thérapie d'inspiration Bobath (thérapie neurodéveloppementale) devenue conventionnelle dans un grand nombre de pays occidentaux depuis 1980, et même dans les pays africains où ces techniques sont encore enseignées actuellement comme référence. Les études de rééducation intensive et de neurorééducation apparaissent vers les années 1990 avec les études de Sunderland [38] et ensuite développées par Kwakkel [33,36,37]. Smith et al [39] sont les premiers à s'intéresser à l'effet de l'intensité dans la rééducation après un AVC en comparant les différentes durées d'un même traitement. Sur 133 hémiparétiques subaigus suivit pendant six mois et répartis en trois groupes : rééducation intensive (augmentation de la durée du travail rééducatif), rééducation conventionnelle et retour au domicile. Les activités de la viequotidienne furent améliorées dès le premier mois de façon importante pour le groupe intensif (1) qui a une tendance à mieux progresser que les deux autres groupes intermédiaires, pour le groupe 2 (conventionnelle) dont les résultats étaient meilleurs que le groupe 3 (retour au domicile) mais restaient néanmoins inférieurs à ceux du groupe 1. De plus il était noté une détérioration plus importante pour certains patients du groupe 3, que dans les groupes 1 et 2, justifiant ainsi l'importance de la rééducation, et de la rééducation intensive en particulier. Par-là, plusieurs approches ont été développées depuis plus de 20 ans : l'entraînementpar des gestes répétitifs et finalisés, support des apprentissages moteurs, est l'un des grands principes utilisés pour la rééducation [11,40]. L'imagerie motrice consiste à répéter mentalement un geste moteur sans exécution réelle. Cette technique permettrait de stimuler les mêmes régions cérébrales que celles impliquées dans la programmation et l'exécution d'un geste par l'effet afférent [41,42]. Dans les deux cas, la rééducation pourrait stimuler les phénomènes de plasticité sensori-motrice. La thérapie par la contrainte associée à l'intensité est basée sur deux principes 
théoriques : (i) lutter contre la sous-utilisation du membre parétique par la contrainte du MS sain ; (ii) favoriser l'extension de la représentation de la main au niveau du cortex moteur primaire (M1) de l’hémisphère lésé par une rééducation intensive du membre parétique [11-13,32-34].

Une revue Cochrane de 33 essais $(n=1853)$ a montré que la pratique répétitive et intense de l'entraînement fonctionnel orienté vers les tâches des MS, était favorable pour les patients hémiparétiques après un AVC par rapport aux soins kinésithérapiques conventionnels. Ces résultats étaient significatifs avec une amélioration très prononcée et l'effet observé dans le groupe répétition intensive était rémanent jusqu'à 6 mois après l'étude [43]. Au-delà de l'intensité et de la répétition, un autre paramètre aussi efficace que les deux précédents est la durée du temps de rééducation ou l'augmentation du nombre de périodes de rééducation [11]. Diverses revues systématiques et méta-analyses ont montré que plus le temps passé en thérapie par l'exercice est important, plus les effets bénéfiques cliniques le sont également. Cette constatation est indépendante du temps d'entraînement ou réentraînement après l'AVC et du type d'intervention de rééducation motrice. Cependant, la dose optimale de thérapie par l'exercice est encore inconnue après un AVC (ceci au regard des profils hétérogènes des patients et du type de lésion). Des méta-analyses systématiques suggèrent qu'un temps de traitement supplémentaire > 16h/mois (4h/semaine) dans les 6 premiers mois après l'AVC donnait des résultats pertinents sur les échelles AVQ, ICF et MIF [37]. De même, Lohse et al [42] ont constaté après la mise en commun de 30 essais ( $\mathrm{n}=$ 1750), qu'il il y avait une corrélation significative entre l'augmentation du nombre d'heures de thérapie physique et l'amélioration des AVQ et de la marche. Ces données suggèrent que des temps d'exercices plus élevés peuvent conduire à de meilleurs résultats après un AVC. Sur la base des méta-analyses et en règle générale, il est recommandé aux patients de faire de l'exercice pendant au moins 45 minutes les jours ouvrables tant qu'il y a des objectifs de rééducation. Car l'intensité augmente l'activation du cortex sensori-moteur et elle est prouvée comme améliorant la performance fonctionnelle du patient, car toutes les études de ces vingt dernières années qui s’y sont intéressées ont conclu significativement sur la supériorité de l'intensité versus technique passive [11,32,3,35].

\section{Discussion, approche clinique et propositions de techniques de rééducation}

\section{1- La question du plateau de rééducation}

Parmi les questions les plus récurrentes en neurorééducation, la question du plateau (période où la récupération stagne) est encore d'actualité. La communauté (médecins, thérapeutes et scientifiques) pense que ce plateau est de 6 mois, et jusqu'à un an pour certains. Mais il se pourrait qu'il y ait une confusion entre la récupération induite par la lésion (plasticité cérébrale) qui dure environ six mois à un an et la plasticité comportementale, qui elle dure toute la vie [11] (figure 6).

Toutefois, nous pensons que le contenu même de la kinésithérapie reste réellement à définir aujourd'hui. 
En pratique quotidienne, le temps de rééducation est bas, ce qui pourrait expliquer la durée du plateau de récupération post-entraînement observé par certaines études [4,5,44,45]. Afin de maintenir le contenu plastique (lié au comportement) après celui impulsé par la lésion, l'entraînement et la modification des comportements moteurs intensifs, répétitifs et maintenus sur une bonne durée, améliorent continuellement les fonctions motrices même 10 ans après la lésion [11,32,34,36-39]. Au regard de la clinique, nous avons observé qu'en rééducation neurologique soit on progresse, soit on régresse, il n'y pas de place pour la stagnation. Un patient qui fait les mêmes exercices avec la même intensité, pendant la même durée temps chaque fois, n'évoluera pas, bien au contraire, il risque de régresser dans le temps. Ce travail intensif dont nous parlons ne doit débuter qu'à distance de l'AVC (en l'absence de contre-indication, une semaine en moyenne après la lésion, et ceci de manière évolutive) et il faut toujours corréler l'intensité du travail donné au patient à son état clinique le jour de la séance [11].

Cependant, dès les premiers jours de l'AVC, un travail de prévention peut être fait au regard de la pathologie musculaire qui est précoce [12-16,18-19] et pouvant entraîner des troubles de la déglutition, une hypoventilation liée au décubitus et au déficit d'amplitude des membres et leur sous-utilisation, une thrombose veineuse profonde, de la constipation, et une atrophie des membres hypo utilisés. Une kinésithérapie de mobilisation active et évolutive au niveau respiratoire, un reconditionnement physique, une mobilisation passive au niveau des MS et MI par des postures de flexion du coude et de rotation externe maximale du bras et élévation-flexion de la hanche sont capitaux pour prévenir ces mécanismes de dégradation musculaire précoces (figure 7). La prise en charge kinésithérapique par un neurorééducateur, ou dans un centre adapté à la neuroréhabilitation est généralement bien tolérée et favorable qu'une rééducation en cabinet de kinésithérapie non spécialisé ou de ville, et le bénéfice est non seulement d'ordre physique, mais aussi psychologique [11,32,46,47].

La bonne prise en charge d'une parésie spastique déformante passe par une évaluation sérieuse, afin de noter objectivement et parfaitement, les gênes et les complications fonctionnelles observées durant un examen clinique lors d'une consultation dédiée. Deux outils peuvent être utilisés : l'échelle de Frenshay modifiéeet l'échelle de Créteil (échelle de Tardieu modifié par l'université de Créteil, CHU Henri Mondor). Ces deux échelles dénommées «Echelle à 5 Etapes » (E5E) de Créteil possèdent une bonne fiabilité inter examinateur et une bonne sensibilité [7,8,9]. L'E5E permet réellement de quantifier la part de rétraction, de la spasticité et la longueur du muscle tout en jaugeant les amplitudes perdues. Pour les cotations musculaires, l'échelle MRC peut servir pendant les premiers jours de la période flasque de l'AVC, mais n'a pas d'utilité lorsque la parésie spastique est déjà installée. Ceci s'explique du fait que la cotation MRC est plus corrélée avec la faiblesse et la gravité et non la spasticité. Or les patients parétiques spastiques sont gênés par leurs antagonistes (co-contraction, hyper activité, dystonie) et pas par la gravité. En outre, leur problème est plus central et périphérique (commande descendante) que périphérique seul. Ce sont les co-contractions qui les empêchent de bien exécuter le mouvement et non la faiblesse du muscle. Lors de l'exécution d'un mouvement, l'antagoniste recrute plus que l'agoniste qui en a plus besoin pour accomplir le mouvement désiré, d'où un déséquilibre de recrutement (figure 4). 


\section{2- Observation et pratique clinique}

Nos patients parétiques spastiques sont généralement vus en phase chronique (> 3mois post-AVC).

La compréhension des caractéristiques d'un entraînement qui conduit à une plasticité cérébrale positive ou délétère «mal adaptative » a largement contribué à développer de nombreuses applications d'optimisation de la performance chez les sujets sains, mais aussi dans la prise en charge des pathologies neurologiques centrales et périphériques, locomotrices et psychiatriques [11]. La première de ces notions est que la simple utilisation d'une fonction l'entretient, d'où le fameux concept anglais («use it or loose it »), voire dans certains cas, l'améliore ( «use it and improve it»). Dans cette optique, les paramètresd'intensité et de répétition associés à la durée de l'entraînement sont primordiaux. Qualitativement, l'attention portée à la tâche, sa spécificité et sa pertinence par rapport aux apprentissages visés représentent également des critères importants dans la récupération motrice post-AVC [48-50] (Figure 8).

Pour qu'un programme d'apprentissage moteur soit efficace, il faut que les exercices proposés soient des mouvements proches de la normale. Les mouvements doivent être orientés et ciblés sur des tâches précises [11,32]. En clinique rééducative, corroborée par l'imagerie médicale, nous savons aujourd'hui que l'imagination d'un mouvement (effet afférent : thérapie miroir, imagination motrice virtuelle, etc.) est moins efficace que l'activation musculaire (efférent : exécution du mouvement en réalité, même si on n'y arrive pas).Il serait mieux d'activer réellement le muscle en question, et que l'attention soit portée sur la tâche à accomplir [51]. Clarson et al [52], dès 1998, insistent sur l'intérêt de nombreuses répétitions. En rééducation, nous insistons sur le programme intensif, soit en termes de difficulté du mouvement travaillé soit par le nombre de répétitions effectuées, associé à une durée assez importante ( $1 \mathrm{~h}$ à $2 \mathrm{~h}$ par jour, pendant au moins 5 jours /semaine, hormis des séances conventionnelles de kinésithérapie) [11] (figures 7 et 9). L'intensité est peut-être le facteur qui a été le moins étudié en imagerie fonctionnelle mais c'est celui qui a été le mieux étudié en termes de clinique simple. Suzuki et al [51] ont comparé la marche d'un patient hémiparétique avec soulagement du poids corporel à une marche sans harnais de soulagement. Ils ont constaté que le fait d'avoir soulagé le poids corporel (par harnais) avait entraîné une diminution de l'activation du cortex sensori-moteur. Dans la même étude [51], deux cadences de marche différentes ont été comparées, et lorsque le patient était forcé à marcher sans harnais, on constatait une amélioration de l'activation du cortex sensori-moteur. De façon similaire et intuitive, lorsqu'on demandait au patient de courir ou d'essayer de courir, on avait une activation supérieure des cortex sensori-moteur et prémoteur.

C'est ça le concept de contrainte et d'intensité $[11,40,42,47,49]$. La contrainte amène à une augmentation des aires et cartes corticales et àune augmentation de leur excitabilité par rapport à l'absence de contrainte. Les sportifs pour augmenter en performance s'entraînent, difficilement, graduellement et pendant de longues périodes de temps avec des contraintes élevées, parfois jusqu'à épuisement physique et même psychique. C'est le même principe d'acquisition de puissance et de compétence physique (plasticité 
musculaire) chez les patients ayant fait un AVC. Car l'entraînement est un tremplin pour une acquisition fonctionnelle associée à une augmentation des cartes corticales pour la mémorisation à long terme $[11,12,13]$. C'est important de comprendre que si on s'abstient de contraindre (volontairement) les patients à un travail de tâches difficiles, actives, répétitives, prolongées et, si on rentre dans une lucidité permanente de travail passif (amusant et juste occupant), alors nous retirons aussi l'excitabilité corticale et par conséquent les résultats efficients. Il ne faut pas que la rééducation soit «marrante » en général, il faut que ce soit difficile et contraignant, soit en augmentant le nombre de répétitions soit en augmentant le temps de rééducation (mais les deux seraient l'idéal) $[11,12,13]$.

\section{3- Proposition de techniques kinésithérapiques dans la rééducation de la parésie spastique déformante}

Les traitements médicamenteux sont utiles pour «ouvrir» des articulations totalement verrouillées afin d'appliquer des techniques physiques par la suite. Nous pouvons citer entre autres les plus employés en clinique. Pour les traitements oraux : baclofène, dantrolène, tizanidine (ils sont peu efficaces si la spasticité est déjà gênante). Le baclofène est utile si la spasticité des membres inférieurs est non gérable par les traitements locaux. Le phénol-alcool est réservé aux nerfs à contingent moteur (nerf obturateur, anse pectorale). Différentes chirurgies peuvent être effectuées (neurotomies périphériques, ténotomies, drezotomies). Pour la spasticité diffuse, on peut utiliser le Sativex®. La toxine botulique est efficace si la gêne est focale ou multifocale, elle agit en périphérique sur les blocs de conduction. Tous ces traitements ont une demi-vie allant de 2 mois à 6 mois. C'est pourquoi les traitements physiques doivent être sollicités pour conserver, voire augmenter les gains acquis durant ces actes médicaux et/ ou chirurgicaux. Les patients ayant eu une lésion neuronale sont dans une situation de déficit cognitivomoteur source d'une hypoactivité auto-imposée, elle même source de réduction de plasticité aggravant la lésion. Nous devons agir sur l'hypoactivité pour briser ce cercle néfaste d'aggravation. Il faut donc utiliser la contrainte dans la difficulté de l'activation musculaire, la répétition, l'intensité, l'attention portée au mouvement afin de promouvoir la plasticité cérébrale et la récupération fonctionnelle induite par le comportement [11]. On sait déjà que l'augmentation de l'intensité de la rééducation est un principe important, mais la kinésithérapie proposée actuellement en cabinet de ville est insuffisante pour avoir une efficacité significative, ceci a été démontré par l'équipe de John Green et al $[44,45]$.

\section{4- Techniques et pratiques}

L'idée du traitement focal est de se concentrer sur un muscle, l'antagoniste, pour en diminuer les résistances passives (rétractions) et actives (co-contractions). L'évaluation permet de sélectionner les muscles antagonistes à traiter [6,7,8,9]. Si l'indice de rétraction est élevé, l'affection musculaire sera traitée par étirement prolongé. Si l'indice de faiblesse/fatigabilité est élevé, l'affection neurologique sera 
traitée par les mouvements alternatifs d'amplitude maximale sans assistance. On se base sur une rééducation centrée sur l'antagoniste à un mouvement donné [9,11,32].

\section{4-A) Techniques et thérapeutiques physiques de l'affection musculaire}

L'étirement est une activité thérapeutique fondamentale de tout thérapeute physique. Un programme d'étirement musculaire est une lutte contre le désordre musculaire de la parésie spastique déformante. L'allongement physique ou la ré-augmentation de l'extensibilité du muscle co-contractant, par des programmes « agressifs » d'étirements quotidiens prolongés, peut diminuer le facteur d'aggravation qui est lamise en jeu des récepteurs à l'étirement du muscle. Un rallongement du muscle hyperactif viendrait en effet réduire la sensibilité des fuseaux neuromusculaires à l'étirement en diminuant leur capacité à aggraver une cocontraction antagoniste exagérée [6-9,11,32]. Le muscle nécessite du temps (au moins 4 mois, pour avoir des premiers résultats significatifs) et de la force pour s'allonger : plus l'étirement dure longtemps (en temps d'application d'exercice et en intensité afin d'aller profondément au-delà des tissus superficiels, et jusqu'à susciter un seuil de douleur tolérable, et y rester minimum pendant 45 secondes, voire une minute en posture étirée), plus il est fort. C'est seulement dans ces conditions que l'on vaincra la parésie spastique déformante (Figure 8) et que le muscle gagnera en longueur $[6,53,54]$. Il faut donc étirer fort et pendant longtemps et y rester à long terme (en allant progressivement). L'étirement doit se prolonger bien après la sortie du patient du centre de rééducation. Pradines [55,56], dans une étude rétrospective, s'est intéressée à un étirement à long terme. La charge et la durée quotidienne sont des paramètres critiques de l'étirement. Pour les muscles qui n'avaient pas été ciblés par le traitement (étirement agressif et chronique, puis auto rééducation guidée), l'indice de rétraction restait globalement stable dans le temps. Concernant les muscles qui avaient été étirés, on a vu leur coefficient de rétraction diminuer quasiment linéairement au cours du temps. Il est donc normal que les études (et les thérapeutes aussi) ayant arrêté leur protocole d'étirements à quelques mois ne montrent que des allongements très peu significatifs. Il faut continuer l'étirement de manière prolongée pour observer des résultats satisfaisants via des arrangement plastiques par afférence en réaction à la boucle gamma. Il existe une corrélation entre la longueur de l'antagoniste et la fonction active contre cet antagoniste et l'équilibre peut être restauré par une mise en tension opposée (étirement de l'agoniste sur l'antagoniste), étant donné que dans les affections dites centrales, ce sont plus les muscles dits ouvreurs qui sont atteints (l'extension est plus difficile que la flexion).

\section{4-B) Techniques et thérapeutiques physiques de l'affection neurologique}

La maladie neurologique a elle aussi ses techniques. On vise à améliorer la plasticité des régions cérébrales intactes. Il faut toujours appliquer le principe d'intensité (répétition + contrainte + augmentation du temps de rééducation). Pour cela on peut mettre en œuvre la technique de l'effort alternatif rapide qui permet de diminuer les co-contractions par l'augmentation de l'inhibition réciproque. 
Cette technique a été testée par Bütefisch [57,58] sur une série d'hémiparétiques dans une période subaiguë à chronique après l'AVC, et ces résultats étaient significatifs sur la commande neurologique (exécution du mouvement volontaire avec réduction des co-contractions et gain d'amplitude articulaire). $\mathrm{Hu}$ et al [59] ont utilisé la technologie des robots pour pratiquer la technique des efforts alternatifs rapides en flexions/extensions du coude auprès de 20 patients lors de 3 à 4 séances par semaine. Ils ont mesuré les niveaux de co-contractions des fléchisseurs et des extenseurs du coude, et ils ont constaté que ces séries d'efforts alternatifs rapides avaient fini par diminuer les niveaux de co-contractions autour de l'articulation du coude. Floeter en 2013 [60] a été plus loin en montrant pourquoi les efforts alternatifs rapides diminuaient les co-contractions autour d'une articulation : l'inhibition réciproque est augmentée par ce type d'entraînement. Un exemple de cible pour mouvement alternatif rapide peut être la poignée de porte, l'interrupteur, et le mouvement maximum (qui peut être marqué par le thérapeute sur un mur). Il faut inscrire le patient dans ce qui est difficile (mais possible) dans ces trois cibles, et y rester en changeant de niveau à chaque fois que le patient atteint le niveau supérieur [13] (figure 8). Les exercices proposés au patient doivent trouver leur prolongement à domicile afin d'être vraiment efficients d'une part, et de briser le cercle néfaste de la sous-utilisation (qui est un inhibiteur majeur de la plasticité) d'autre part. De ce fait, le contrat d'auto-rééducation guidée (CAG) [61] doit être initié via un registre (commun accord du patient et du thérapeute) où le patient doit noter après chaque réalisation les thérapeutiques physiques prescrites par le thérapeute à réaliser à domicile. Le registre est une technique psychologique d'importance primordiale, car il permet un renforcement positif [11], c'est-à-dire que les notes prises la veille incitent à faire mieux le jour suivant. Le registre doit être tenu par le patient lui-même dans la mesure du possible afin d'obtenir cet effet «antidépresseur» et motivationnel. Cela va permettre au patient de mettre en pratique les techniques d'auto-entraînement à long terme. Si l'on combine la technique psychologique du registre aux techniques physiques d'auto-étirements prolongés et d'efforts alternatifs rapides, on aboutit à un système de travail efficace, tant pour le patient, que pour le thérapeute. Car nous constatons que la kinésithérapie seule ne peut pas résoudre le problème de la parésie spastique déformante [44,45], c'est pourquoi l'initiation d'une formation d'autorééducation guidée (patients) est désormais urgente pour tout rééducateur afin d'espérer quantifier les gains thérapeutiques.

\section{5- Mécanismes physiologiques et biologiques des deux techniques (étirement agressif, prolongé et mouvement alternatif à haute amplitude) proposées}

Au niveau des mécanismes physiologiques, l'entraînement en efforts alternatifs rapides d'amplitude maximale et non assistés diminue les co-contractions autour d'une articulation par restauration de l'inhibition réciproque. La difficulté, la rapidité de contraction sont des éléments excitateurs et créateurs des cartes corticales et augmentent par ailleurs l'excitation des forces de connexion synaptique $[8,9,11,32,60,62,63]$. 
$\mathrm{Au}$ niveau des mécanismes biochimiques, les mouvements spontanés créent aussi du flux au niveau extracellulaire (glucose, acides aminés, neuro-actifs (ASP, taurine), etc.). La sous- utilisation, au contraire, baisse le taux extracellulaire de glucose et même la qualité d'oxygène et du flux circulatoire menant à la mort neuronale $[8,9,11]$.

L'entraînement en efforts alternatifs rapides d'amplitude maximale et l'étirement « agressif » chronique prolongés au domicile sont des thérapeutiques physiques induisant la plasticité cérébrale. Ces mouvements alternatifs rapides doivent être d'amplitude maximale pour ceux qui parviennent à créer un mouvement car cela induit un étirement excentrique de l'antagoniste qui s'ajoute à l'effort actif dans la lutte des co-contractions, les mouvements actifs non assistés et répétés en vitesse maximale pour que la contrainte imposée au SNC soit maximale. Cet entraînement doit être proposé de la même manière aux patients qui présentent initialement un mouvement inverse à celui recherché (co-construction de l'antagoniste supérieure à l'agoniste lors de l'exécution d'un mouvement) en séries courtes car l'épuisement à la fois psychique et neuronal apparaît rapidement. Après un certain temps d'entraînement, on pourra voir le mouvement se neutraliser puis devenir positif. L'entraînement moteur intensif des muscles les moins hyperactifs devrait perturber le cycle de sous-utilisation spastique, et l'utilisation concomitante d'agents agressifs d'étirement et d'affaiblissement focal dans leurs antagonistes plus hyperactifs et raccourcis, devrait briser le cycle de suractivité-contracture-suractivité [8,9,11]. La fatigue doit être visée à chaque entraînement, car la fatigue est le produit stimulateur de croissance de la plasticité cérébrale et musculaire. C'est le même processus de plasticité que chez les sportifs, plus ils s'entraînent difficilement jusqu'à la fatigue (épuisement), plus leur organisme devient tolérant aux activités physiques qui paraissaient difficiles antérieurement. Ça ne marche que si on fait un peu plus qu'hier ou la semaine dernière [11].

\section{Conclusion}

La spasticité est un symptôme très fréquent dans la plupart des affections du SNC. Elle nécessite de répertorier scrupuleusement les gênes et les freins qu'elle peut induire. Les gênes potentielles sont nombreuses, et les freins aussi sont variables en fonction des situations de vie quotidienne. Elles sont également évolutives ou régressives dans le temps (en fonction de l'entraînement ou de l'inactivité). Elles sont focales ou diffuses dans la majoritédes cas. Une fois identifiées, l'étape suivante consiste à déterminer la stratégie thérapeutique (après une évaluation à 5 étapes avec l'échelle de Créteil qui est plus quantitative et qualitative, car elle permet de savoir effectivement le degré de spasticité et surtout sa nuisance dans l'accomplissement du mouvement actif) issue de l'évaluation clinique. La récupération fonctionnelle de la parésie spastique déformante est un processus hétérogène, complexe, long et multidisciplinaire (au regard même de sa chronicité et de sa déshabituation à l'emploi chronique du membre parétique) qui se produit par une combinaison de récupération neurologique spontanée (plasticité cérébrale : induite par la lésion ellemême) et d'un processus d'apprentissage moteur, y compris la semi restitution (la fonctionnalité du tissu neuronal endommagé), la substitution (réorganisation de voies neuronales pour réapprendre les fonctions 
perdues), et la compensation (amélioration de la disparité entre les capacités affaiblies d'un patient et les exigences de leur environnement) [11]. Cependant, à côté de ces mécanismes sous-jacents, il y a bien des preuves qu'un entraînement moteur intensif, spécifique (tâches orientées) au contexte et à la tâche répétée donne une satisfaction au-delà des niveaux attendus de récupération spontanée (plasticité post-lésionnelle). Une bonne récupération en neurorééducation ne veut pas dire récupération ou arrangement total. L'entraînement en efforts alternatifs rapides d'amplitude maximale et non assistés diminue les cocontractions autour d'une articulation, par restauration de l'inhibition réciproque, et l'étirement agressif chronique prolongé au domicile sont les deux traitements qui influencent directement sur la plasticité cérébrale avec la possibilité de mise en place de Contrat d'Autorééducation Guidée (CAG). Il faut donc demander aux patients des efforts alternatifs rapides qui donnent parfois des mouvements lorsquele patient a un bon niveau. Ces mouvements alternatifs rapides doivent être d'amplitude maximale pour ceux qui parviennent à créer un mouvement, car cela induit un étirement excentrique de l'antagoniste qui s'ajoute à l'effort actif dans la lutte des co-contractions. Le registre d'autorééducation guidé doit être bien maintenu par le patient et vérifié à chaque rendez-vous par le thérapeute qui pourra, après une évaluation, changer les muscles à étirer à domicile. Il est conseillé de ne pas donner au patient plus de 4 muscles à étirer à domicile, de plutôt insister sur un petit nombre de muscles et d'augmenter le temps de rééducation à domicile qui est souvent de 45 min à $2 \mathrm{~h}$ par jour [11], et de répartir entre les muscles et les entrainements excentriquesà intensité maximale. Le thérapeute doit revoir le patient 2 à 3 fois par semaine. 


\section{Remerciements :}

CHU Henri Mondor et Albert Chenevier, en particulier le service du Pr Jean Michel Gracies, laboratoire analyse et restauration $\mathrm{du}$ mouvement \& rééducation neurolocomotrice et ostéoarticulaire pour leur engagement dans le traitement physique de la parésie spastique déformante, et une reconnaissance pour toute l'équipe soignante, médecins et thérapeutes.

CHU Rémond Poincaré, Garches (université de Versailles, Paris 13 ; en particulier le service de médecine physique et réadaptation (MPR) de Netter, service du Pr François Genet, et le laboratoire handicap neuromusculaire, physiopathologie, biothérapie et pharmacologie appliquées, ENDINCAP, U1179, INSERM.

La faculté de médecine Sorbonne université (Paris VI, Pierre et Marie Curie), et ses unités Trans Innove Longévité (TIL), gérontologie/gériatrie et ses hôpitaux universitaires Pitié-Salpêtrière et Charles Foix. Et tous les collègues professeurs, assistants qui ont contribué de près ou de loin à cet article.

Laboratoire de recherche des neurosciences de la faculté des sciences de la santé et de psychologie de Bircham International University, Madrid, Espagne.

Institut des neurosciences appliquées et de rééducation appliquée (INAREF), et ses structures Neureab et APARC.

Déclaration de liens d'intérêts : Les auteurs déclarent ne pas avoir de liens d'intérêts. 


\section{Référence}

1- Feigin VL, Forouzanfar MH, Krishnamurthi R, et al. Global and regional burden of stroke during 1990-2010: findings from the global burden of disease study 2010. Lancet 2014; 383:245-54.

2- Lackland DT, Roccella EJ, Deutschet AF, et al. Factors influencing the decline in stroke mortality: a statement from the American Heart Association/American Stroke Association. Stroke J Cereb Circ $2014 ; 45: 315-53$.

3- HAS. Accident vasculaire cérébral. Pertinence des parcours de rééducation/réadaptation après la $\begin{array}{lllll}\text { phase } & \text { initiale } & \text { mai } & 2019 . & \text { https://has- }\end{array}$ sante.fr/plugins/ModuleXitiKLEE/types/FileDocument/doXiti.jsp?id=c_2972909 [Consulté le 16 mars 2021].

4- Jorgensen HS, Nakayama H, Raaschou HO, et al. Outcome and time course of recovery in stroke. Part I: Outcome the Copenhagen Stroke Study. Arch Phys Med Rehabil 1995;76(5):399-405.

5- Jorgensen HS, Nakayama H, Raaschou HO, et al. Outcome and time course of recovery in stroke. Part II: Time course of recovery. The Copenhagen Stroke Study. Arch Phys Med Rehabil 1995;76(5):40612.

6- Vinti M, Couillandre A, Hausselle J, et al. Influence of effort intensity and gastrocnemius stretch on co-contraction and torque production in the healthy and paretic ankle. Clin Neurophysiol 2013;124(3):528 35 .

7- Gracies JM. Pathophysiology of spastic paresis. Part I. Paresis and soft tissue contracture. Muscle Nerve 2005;31(5):535-51.

8- Gracies JM. Pathophysiology of spastic paresis. Part II. The emergence of muscle overactivity. MuscleNerve 2005;31(5):552-71.

9- Gracies JM. Coefficients of impairment in deforming spastic paresis. Ann Phys Rehabil Med 2015;58(3):173-8.

10- Meige H. Les amnésies motrices fonctionnelles et le traitement des hémiplégiques. Rev Neurol 1905 ; $13: 183-4$.

11-Npochinto Moumeni I. Plasticité cérébrale : régénération ? réparation ? réorganisation ? ou compensation ? Que savons-nous aujourd'hui ? Neurol Psychiatr Gériatr 2020, https://doi.org/10.1016/j.npg.2020.11.002

12- Taub E, Berman A. Movement and learning in the absence of sensory feedback in the neuropsychology of spatially oriented behavior. Homewood: Dorsey Press, 1968: 173-92.

13- Knapp HD, Taub E, Berman AJ, et al. Effect of deafferentation on a conditioned avoidance response. Science 1958; 128: 843-3.

14- Tardieu C, Huet TE, Tabary JC. Diminution de l'extensibilité musculaire et réduction du nombre de sarcomères dans le muscle soleus suite à une injection locale de toxine tétanos. J Neurol Sci 1979;40(2-3):123-31.

15- Tabary JC, Tardieu C, Tardieu G, et al. Changements physiologiques et structurels dans le muscle soleus du chat dus à l'immobilisation à différentes longueurs par des plâtres. J Physiol 1972; 224(1): 231-44.

16-Gioux M, Petit J. Effects of immobilising the cat peroneus longus muscle on the activity 314 of its ownspindles. J Appl Physiol 1993;75:2629-30.

17-Giroux-Metges MA, Pennec JP, Petit J, et al. Effects of immobilizing a single muscle on the morphologyand the activation of its muscle fibers. Exp Neurol 2005;194 : 495-505. 
18- Sherrington CS. Decerebrate rigidity, and reflex coordination of movements. J Physiol 1898; 22(4):319-32.

19- Babinski J. Contracture tendino-reflexe et contracture cutanéo-reflexe. Rev Neurol 1912; 14:77-80.

20- Tardieu G, Ronddt P, Tabary J.-C. Essai de classification des raideurs musculaires d'origine cérébrale. Recherche d'un mode d'appréciation des thérapeutiques. Revue Neurol 1957 ; 97 (4) : 264-75.

21-Lance JW. Symposium synopsis. In: Feldman RG, Young RR, Koella WP, Spasticity: disordered motor control. Symposia Specialists, Miami, 1980: 485-94.

22- Von Monakov C. Die Lokalisation im Grosshim und der abbauder funktion durch kortikale herde. Wiesbaden: Verlag Berman;1914.

23- Tower SS. Pyramidal lesion in the monkey. Brain 1940; 63: 36-90.

24- Jalal N, Gracies JM, Zidi M. Mechanical and microstructural changes of skeletal muscle following immobilization and/or stroke. Biomech Model Mechanobiol 2020; 19:61-80.

25- Taub E, Miller NE, Novack, et al. Technique to improve chronic motor deficit after stroke. Arch Phys Med Rehabil 1993;74:347-54.

26- Taub E, Uswatte G, King DK, et al. A placebo-controlled trial of constraint-induced movement therapy forupper extremity after stoke. Stroke 2006; 37(4): 1045-9.

27-Wolf SL, Winstern Cj, Taub E, et al. Effect of constraint induced movement therapy on upper extremity function 3 to 9 months after stroke: The EXCITE randomized clinical trial. JAMA 2006; 296: 2095-104.

28- Van Der Lee JH, Beckerman H, Lankhorst GJ, et al. Constraint-induced movement therapy. Arch Phys Med Rehabil 1999; 80: 1606-7.

29- Baron JC, Cohen LG, Crammer S, et al. Neuroimagine in stroke recovery: a position paper from the first international workshop on neuroimaging and stroke recovery. Cerebrovasc Dis 2004; 26018 : 260-7.

30- Ostendorf CG, Wolf SL. Effect of forced use of the upper extremity of a hemiplegic patient on changes in function. A single-case design. Phys Ther 1981; 61: 1022-8.

31- Van Der Lee JH, Beckerman H, Lankhorst GJ, et al. Constraint-induced movement therapy. Arch Phys Med Rehabil 1999; 80: 1606-7.

32- Meimoun M, Bayle N, Baude M, et al. Intensité et rééducation motrice dans la parésie spastique. Rev Neurol 2015;171(2):130-40.

33- Kwakkel G, Wagenaar RC, Koelman TW, et al. Effects of intensity of rehabilitation after stroke. A research synthesis. Stroke 1997;28(8):1550-6.

34- Sterr A, Elbert T, Taub E, et al. Longer versus shorter daily constraint-induced movement therapy of chronic hemiparesis: and exploratory study. Arch Phy Med Rehabil 2002; 83: 1374-7.

35- Bobath B. Observation on adult hemiplegia and suggestions for treatment. Physiotherapy 1959;45:279- 89.

36- Kwakkel G, Wagenaar RC, Twisk JW, et al. Intensity of leg and arm training after primary middlecerebral-artery stroke: a randomised trial. Lancet 1999;354(9174):191-6.

37- Kwakkel G, van Peppen R, Wagenaar RC, et al. Effects of augmented exercise therapy time after stroke: a meta-analysis. Stroke 2004;35(11):2529-39.

38- Sunderland A, Tinson DJ, Bradley EL, et al. Enhanced physical therapy improves recovery of arm function after stroke. A randomised controlled trial. Neurol Neurosurg Psychiatry 1992; 55(7): 530- 
5.

39- Smith MC, Byblow WD, Barber PA, et al. Proportional recovery from lower limb motor impairment after stroke. Stroke 2017;48(5):1400-3.

40- Npochinto Moumeni I, Mourey F. Intérêt en EHPAD du robot émotionnel Pepper dans les troubles neurocomportementaux de la maladie d'Alzheimer. Neurol Psychiatr Gériatr $2021 ; 21(121)$ :11-8.

41- Lotze M, Braun C, Birbaumer N, et al. Motor learning elicited by voluntary drive. Brain 2003;126(Pt 4):866-72.

42- Lohse $\mathrm{K}$, Lang C, Boyd L, et al. Is more better? Using metadata to explore dose-response relationships in stroke rehabilitation. Stroke 2014; 45(7): 2053-8.

43- French B, Thomas LH, Coupe J, et al. Repetitive task training for improving functional ability after stroke. Cochrane Database Syst Rev 2016; 11(11):CD006073.

44- Green J, Forster A, Bogle Sue, et al. Physiotherapy for patients with mobility problems more than 1 year after stroke: a randomised controlled trial. Lancet 2002;359(9302):199-203.

45- Green J, Young J, Forster A, et al. Combined analysis of two randomized trials of community physiotherapy for patients more than one year post stroke. Clinic Rehab 2004;18(3):249-52.

46- Veerbeek J, van Wegen E, Kwakkel G, et al. Is accurate prediction of gaitin non-ambulatory stroke patients possible within 72 hours poststroke? The EPOS study. Neurorehabil Neural Repair 2011;25(3):268-74.

47- Veerbeek J, van Wegen E, van Peppen R, et al. What is the evidence for physical therapy poststroke? A systematic review and meta-analysis. PLoS One 2014; 9(2): e87987.

48- Bach-y-Rita P, Kaczmarek KA, Tyler ME, et al. Form perception with a 49-point electro tactile stimulus array on the tongue: a technical note. J Rehabil Res Dev 1998;35(4):427-30.

49- Bach-y-Rita P. Central nervous system lesion: Sprouting and unmasking in rehabilitation. Arch Phys Med Rehab1981; 62:413-7.

50- Katzman R, Björklund A. Evidence for regenerative axon sprouting of central catecholamine neurons in the rat mesencephalon following electrolytic lesions. Brain Res 1971; 25:579—96.

51-Suzuki M, Mihai I, Ono T, et al. Prefrontal and premotor cortices are involved in adapting walking end running speed on the treadmill: and optical imaging study. Neuroimage 2004: 23(3): 1020-6.

52- Classen J, Liepert J, Hallett M, et al. Rapid plasticity of human cortical movement representation induced by practice J Neurophysiol $1998 ; 79(2): 1117-23$.

53- Moriyama H, Tobimatsu Y, Ozawa J, et al. Amount of torque and duration of stretching affects correctionof knee contracture in a rat model of spinal cord injury. Clin Orthop Relat Res 2013;471:3626-36.

54- Ada L, Goddard E, McCully J, et al. Thirty minutes of positioning reduces the development of shoulder external rotation contracture after stroke: a randomized controlled trial. Arch Phys Med Rehabil 2005;86(2):230-4.

55- Pradines M, Portero R, Gracies JM, et al. Muscle lengthening and structural changes in the muscletendon complex of triceps surae after 1 year of rehabilitation including a daily self-stretch program in patients with chronic hemiparesis. Ann Phys Rehabil Med 2016;59S:e73. 
56- Pradines M, Baude M, Jean MG, et al. Effect on passive range of motion and functionalcorrelates after a long-term lower limb self-stretch program in patients with chronic spastic paresis. PM R 2018;10(10):1020-31.

57- Bütefisch CM, Davis BC, Wise SP, et al. Mechanisms of use dependent plasticity in the human motorcortex. Proc Natl Acad Sci U S A 2000;97:3661-5.

58- Bütefisch C, Hummelsheim H, Denzler P, et al. Repetitive training of isolated movements improves the outcome of motor rehabilitation of the centrally paretic hand. J Neurol Sci 1995;130(1):59-68.

59- Hu X, Tong KY, Song R. Variation of muscle coactivation patterns in chronic stroke during robotassisted elbow training. Arch Phys Med Rehabil 2007;88:1022-9.

60- Floeter MK, Danielian LE, Kim YK, et al. Effects of motor skill learning on reciprocal inhibition. Restor Neurol Neurosci 2013;31(1):53-62.

61- Gracies JM. Guided self-rehabilitation contract in spastic paresis. Springer International Publishing, Switzerland, 2016.

62- Moseley AM, Lanzarone S, Bosman JM, et al. Ecological validity of walking speed assessment after traumatic brain injury: a pilot study. J Head Trauma Rehabil 2004;19(4):341-8.

63- Kandel ER, Klein M, Castellucci VF, et al. Some principles emerging from the study of short-and long-term memory. Neurosci Res 1986;3(6):498-520. 
Tableau 1 : Gêne fonctionnelle et design moteur socio comportemental induit par la spasticité.

La désocialisation (gêne et exclusion sociale) et la demande d'adaptation à ce nouveau modèle de corps imposé nécessitent aussi l'intervention du psychologue et une bonne assistance (soutien) familiale et amicale. Car l'acceptation de ce trouble corporel est parfois très difficile à accepter

\begin{tabular}{|l|l|}
\hline MEMBRE SUPERIEUR & MEMBRE INFERIEUR \\
\hline Difficultés de nursing & Manoeuvre de triple flexion \\
\hline Douleurs spontanées ou provoquées & $\begin{array}{l}\text { Désadaptation posturale et perte d'équilibre à h } \\
\text { moindre surprise }\end{array}$ \\
\hline Rétraction ostéotendineuse & Modification de schéma de la marche \\
\hline $\begin{array}{l}\text { Difficulté d'accès à la paume de la main et/ou au plis } \\
\text { du coude }\end{array}$ & Centre de pesanteur et de pression axiale modifié \\
\hline Macération et lésion cutanée & $\begin{array}{l}\text { Varus équin gênant à la phase du simple et double } \\
\text { appui }\end{array}$ \\
\hline Difficulté d'habillage & Griffe d'orteil douloureuse, précisément de l'hallus \\
\hline Perturbation de l'activité de préhension & Clonus et loge gênant à la marche \\
\hline
\end{tabular}

\begin{tabular}{|l|l|}
\hline Action délétère sur l'équilibre et la marche & Adduction de cuisse perturbant l'accès au périnée \\
\hline Clonus du membre supérieur au moindre effort & Attitude en triple retrait \\
\hline Désocialisation et affection psycho exclusive & Déshabituation à l'effort de la marche \\
\hline $\begin{array}{l}\text { Démotivation aux tâches bimanuelles ou devant } \\
\text { inclure juste le bras lésé }\end{array}$ & Dystonie de l'hallus en extension \\
\hline Restriction de certaines activités de la vie & Survenue d'escarre \\
\hline Perte de son emploi & $\begin{array}{l}\text { Diminution de la motricité et hyper extension du } \\
\text { membre lésé (triple extension) }\end{array}$ \\
\hline Perte de son élégance physique (membre déformé) & Perte de sa marche et élégance de sa motricité \\
\hline
\end{tabular}




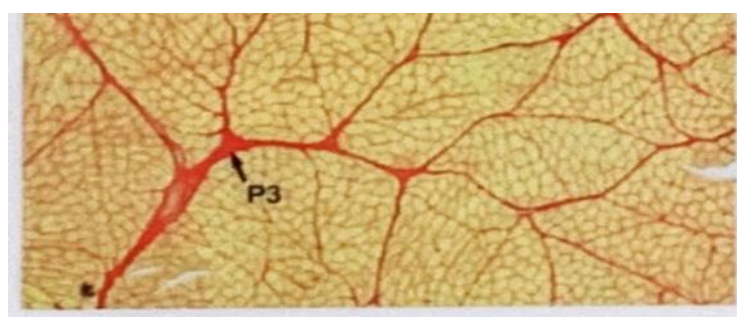

Muscle sain

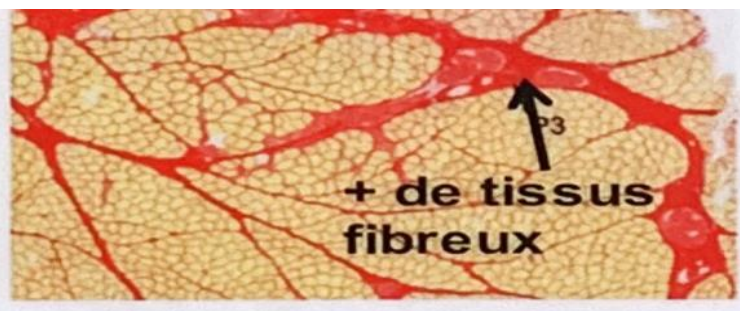

Muscle rétracté

Figure 1 : Muscle sain et muscle rétracté ou spastique car sous-utilisé.

On constate la dégradation de la microstructure et des composantes biologiques, Ubiquitin-proteasome et une augmentation du taux collagène extrasarcomérique, transformant ainsi les caractéristiques des fibres I en fibres II, tout en transformant le tissu musculaire endommagé en tissu graisseux, non extensible qui va créer l'hyperactivité. Le clinicien dans son examen quotidien, ne verra lui qu'un muscle raccourci, hyper actif, fatigable, un muscle rigidifié, et un clonus au moindre effort, et parfois même un mouvement inversé induit par la co-contraction lors de l'effort du patient à faire un mouvement. 


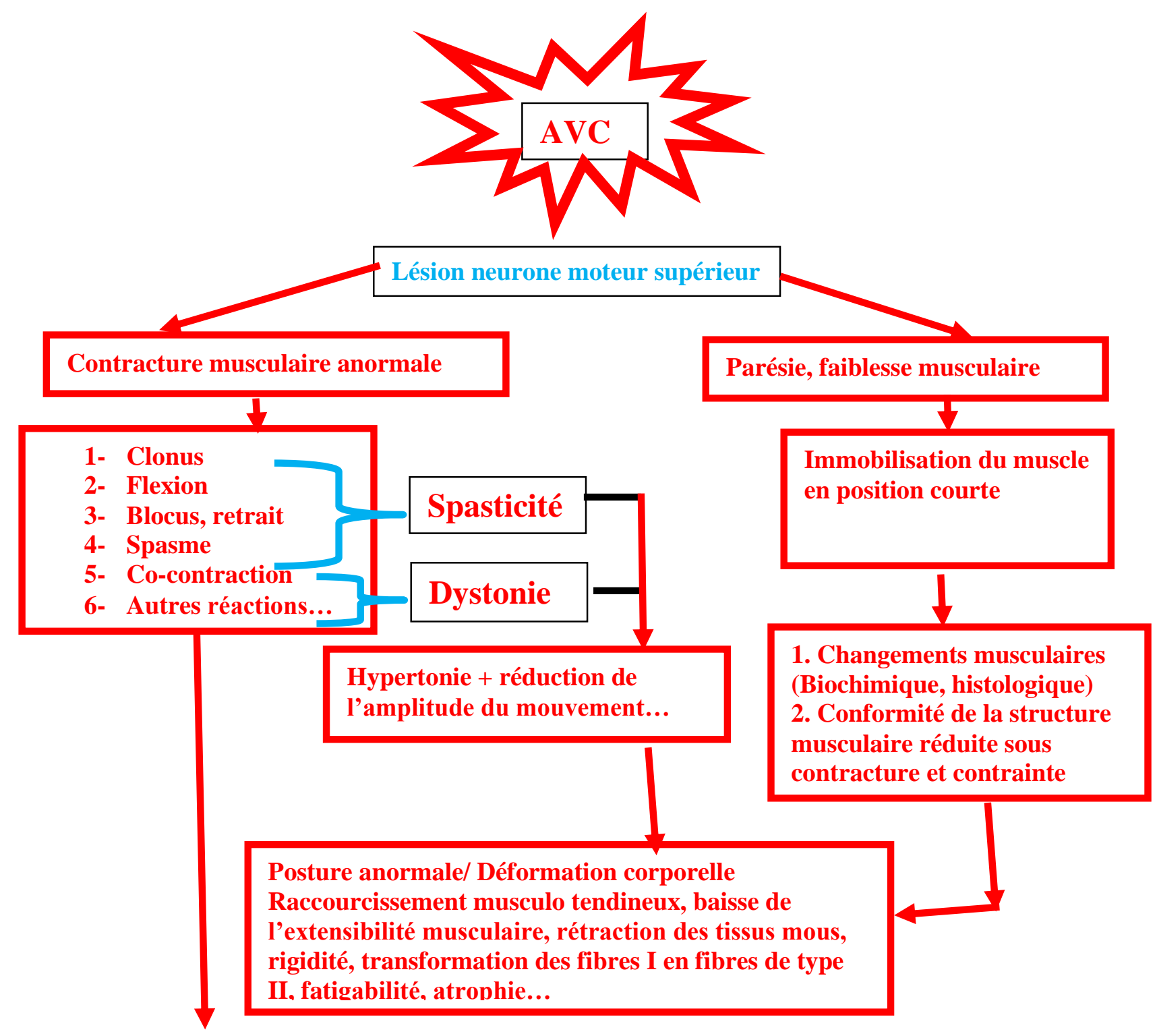

Fonction altérée $\rightarrow$ limitation d'activité $\rightarrow \underline{\text { Situation de handicap }}$

Figure 2 : Neurophysiologie de la parésie spastique déformante, de la lésion (AVC) au handicap. 


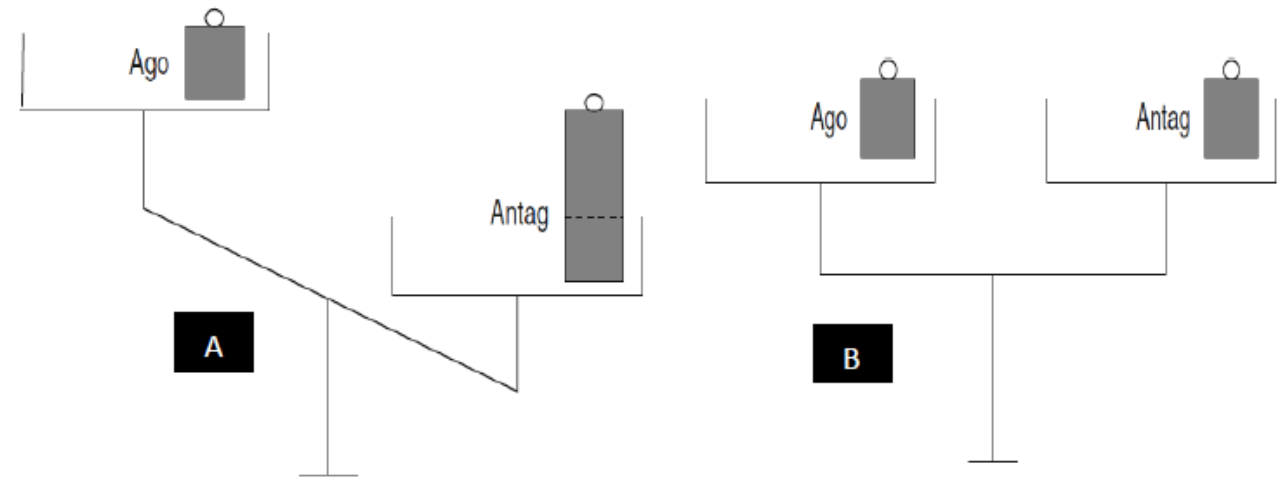

Figure 3 : Déséquilibre intermusculaire.

A : rétraction de l'Ago (agoniste) et tension de l'Antag (antagoniste), l'antagoniste est le véritable problème chez les hémiparétiques ; B : équilibre souhaité entre A et B. 

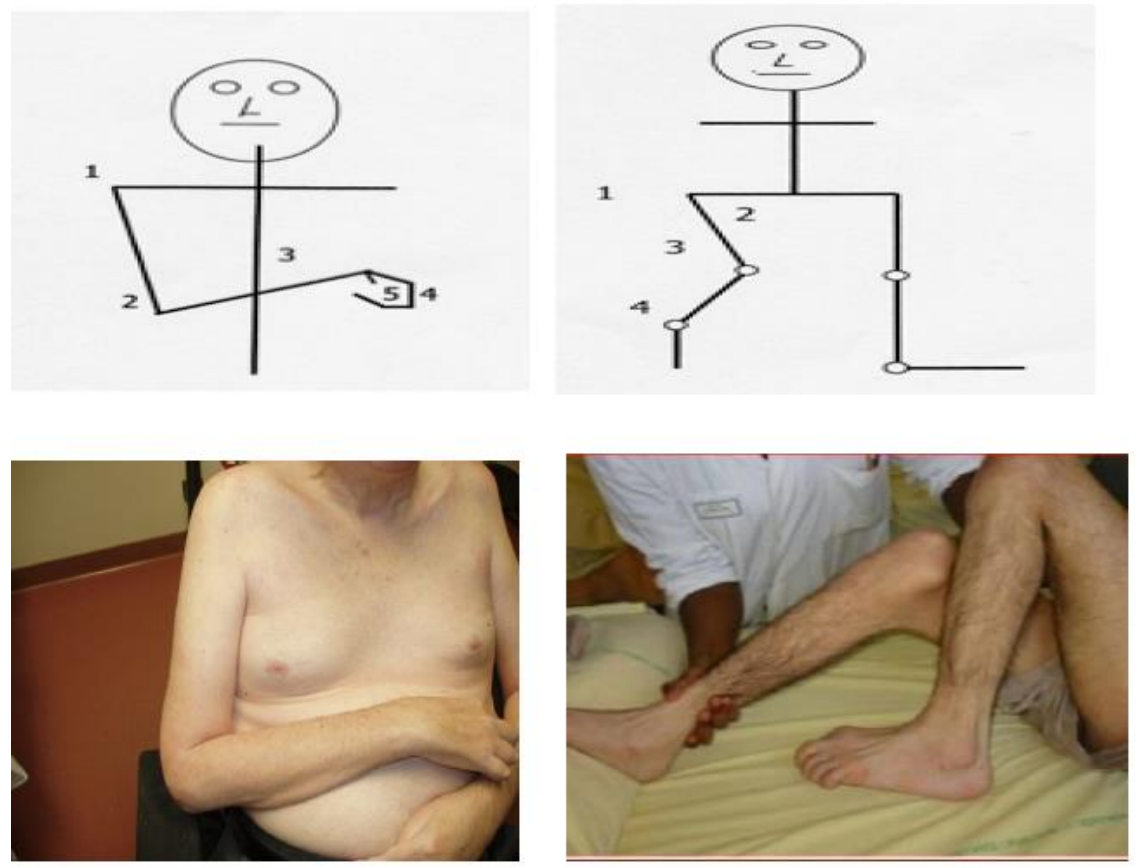

Figure 4 : Schéma et photographies représentant le type de rétractions observées au membre supérieur (à gauche) et au membre inférieur (à droite) chez le patient hémiparétique spastique. 

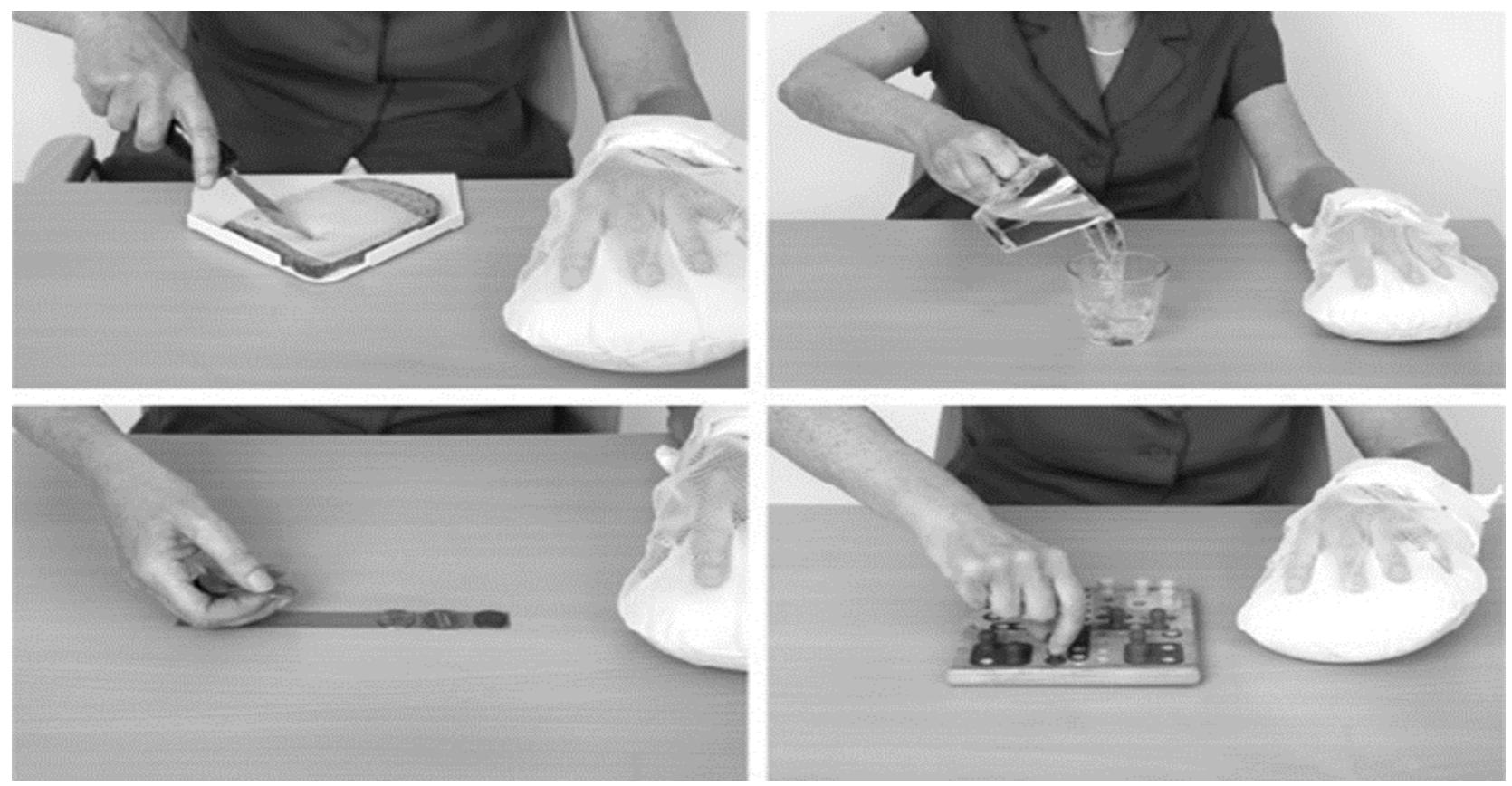

Figure 5 : Exemple de thérapie par contrainte induite (immobilisation du membre sain). 


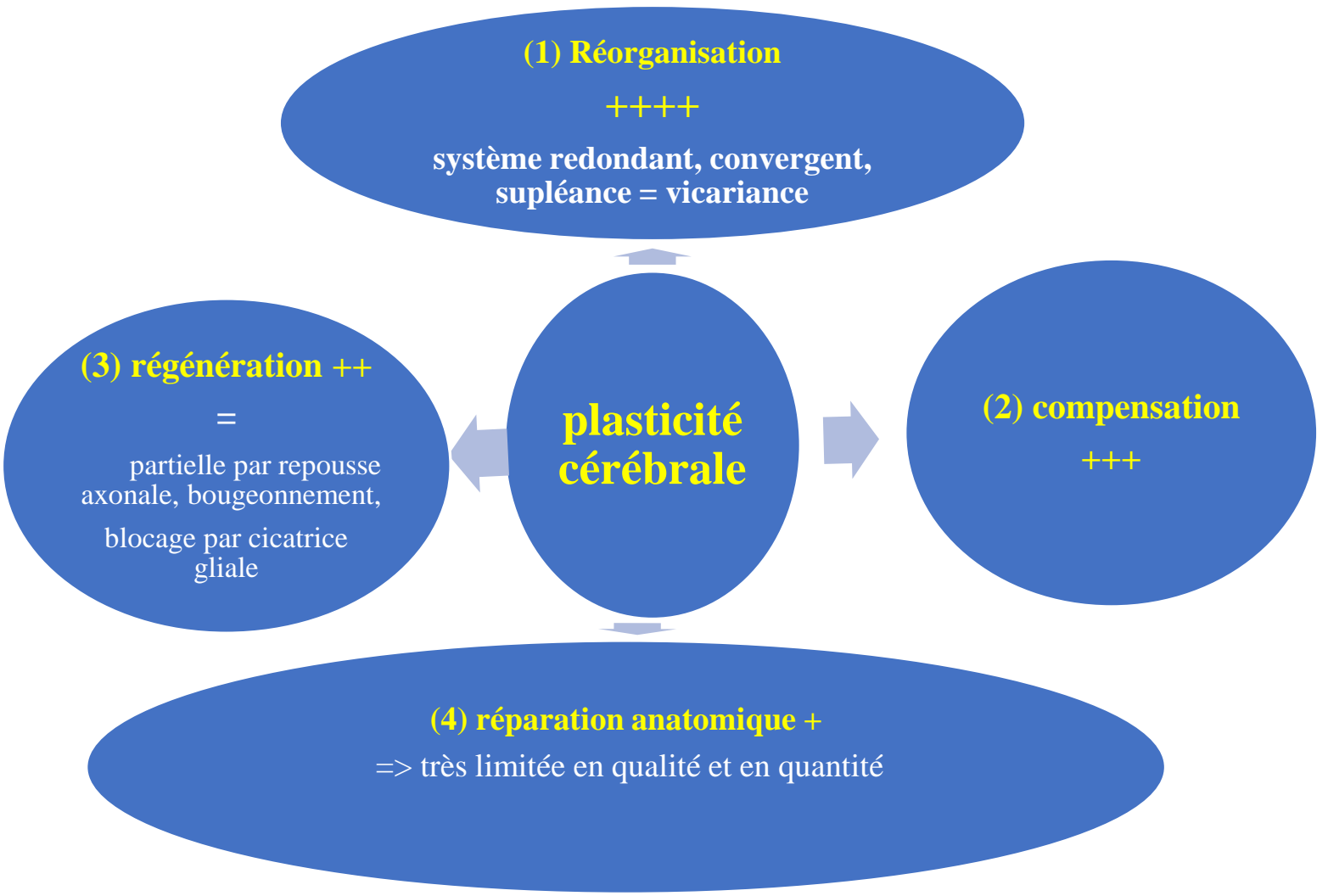

Figure 6 : Présentation des arrangements cérébraux (post-lésion) connus à ce jour par ordre de fréquence (de ++++ à +) et de réalisation.

La neurorééducation (en quantités et en spécificités de techniques) trouve sa place autour de ces quatre pôles afin de favoriser le maximum de récupération (d'après [11]). 

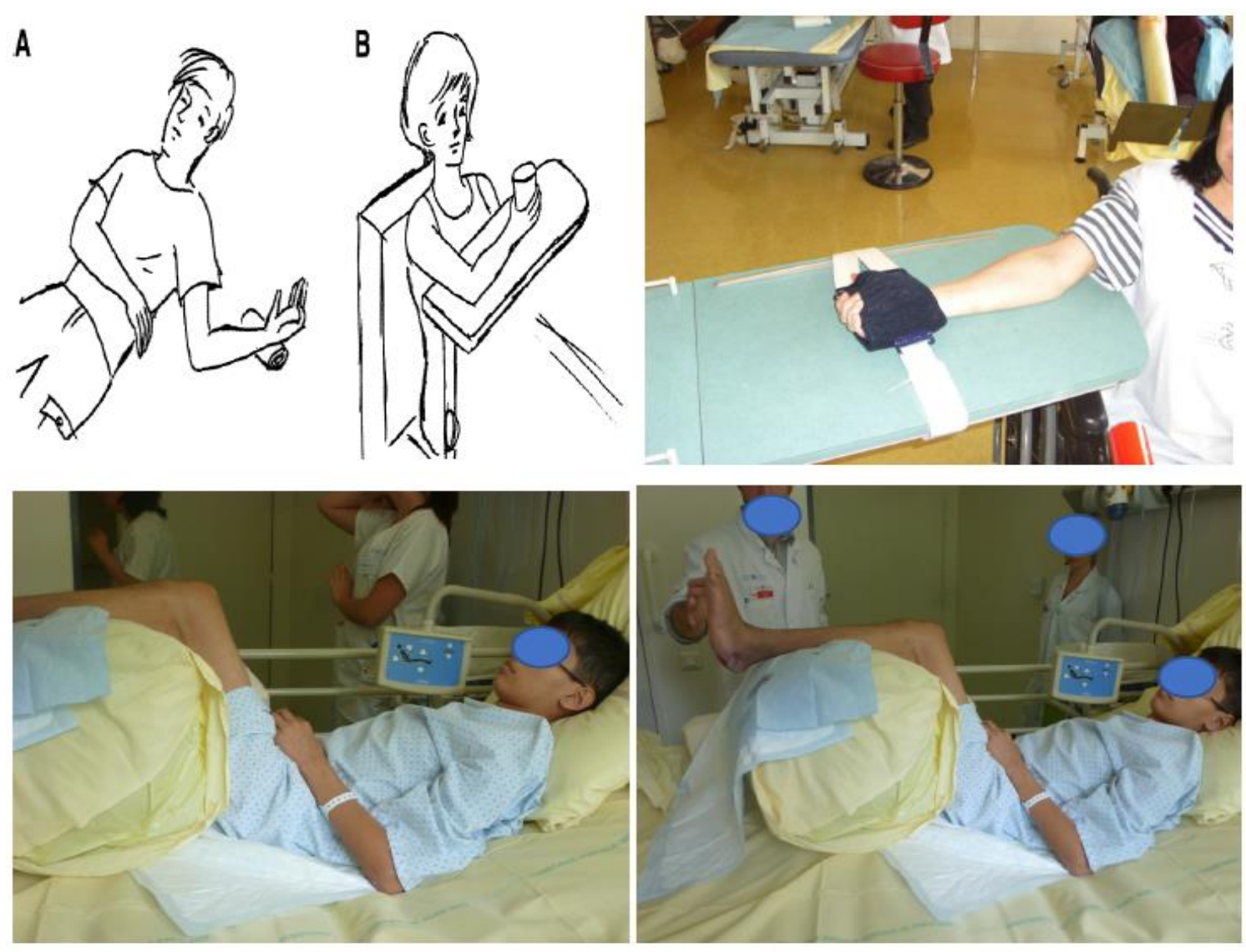

Figure 7 : Différentes postures à mettre en place dès les premières heures en service neurovasculaire. 


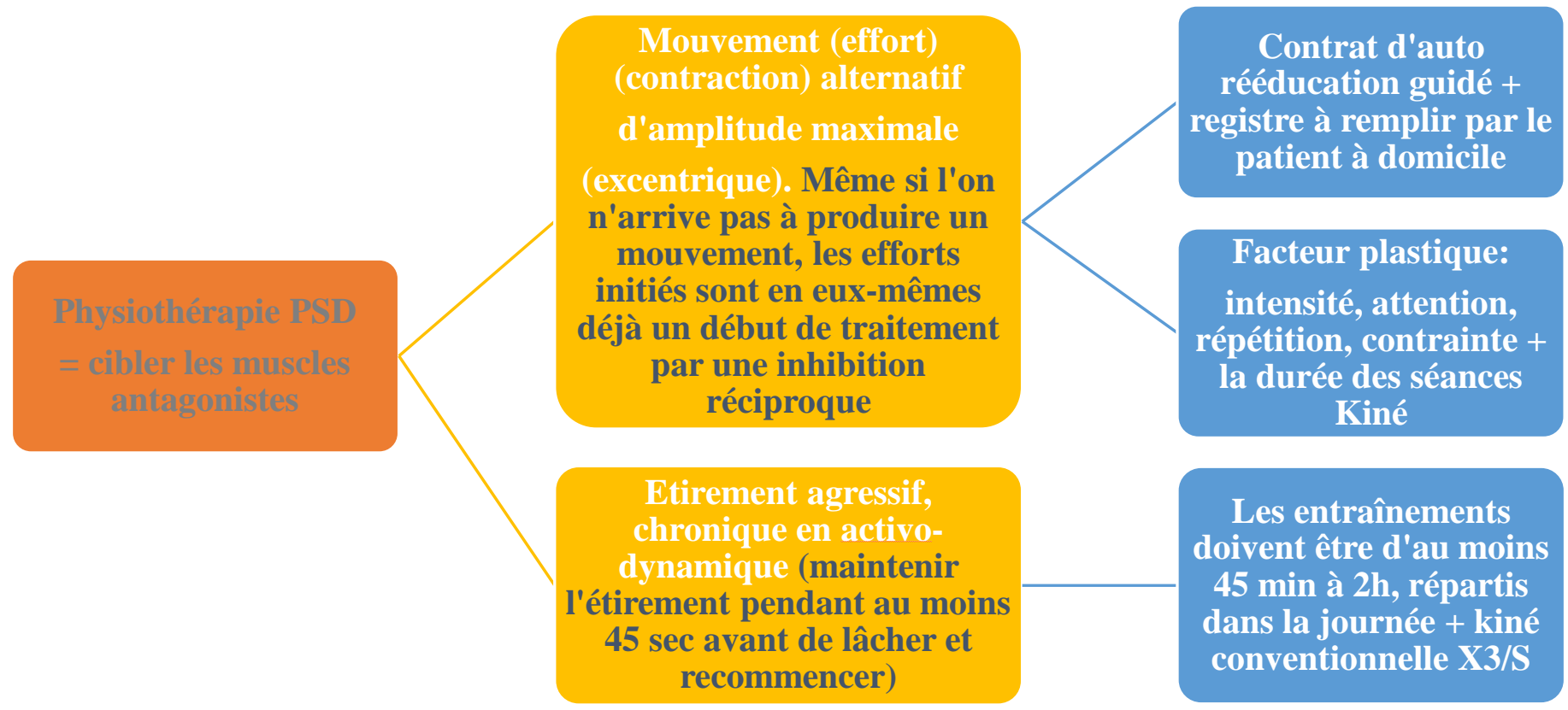

Figure 8 : Physiothérapie de la parésie spastique déformante (PSD). 


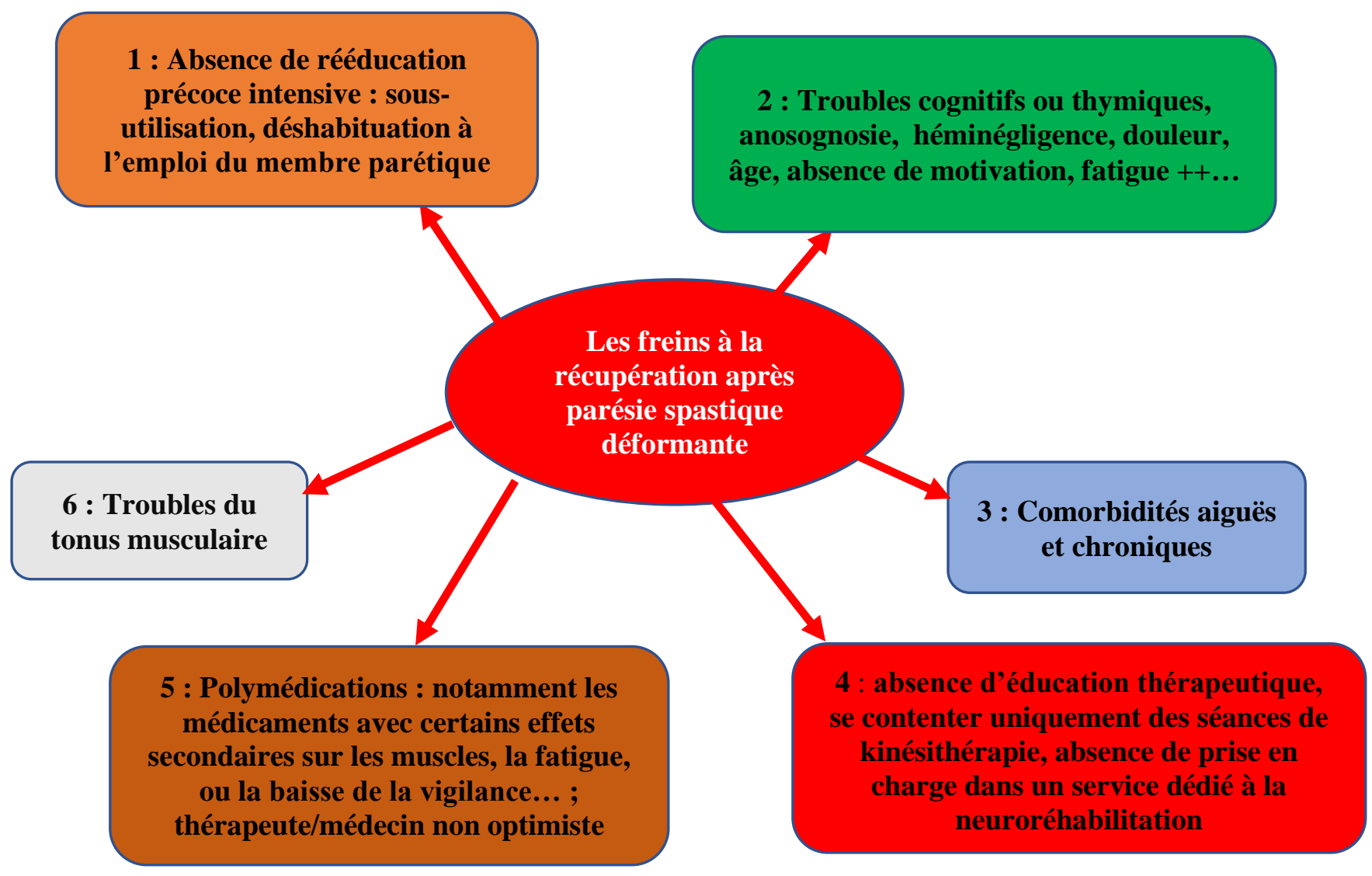

Figure 9 : Exemples de quelques freins à la récupération fonctionnelle post-parésie spastique. 